\title{
IRS-Assisted Secure UAV Transmission via Joint Trajectory and Beamforming Design
}

\author{
Xiaowei Pang, Nan Zhao, Senior Member, IEEE, Jie Tang, Senior Member, IEEE, Celimuge Wu, Senior \\ Member, IEEE, Dusit Niyato, Fellow, IEEE, and Kai-Kit Wong, Fellow, IEEE
}

\begin{abstract}
Despite the wide utilization of unmanned aerial vehicles (UAVs), UAV communications are susceptible to eavesdropping due to air-ground line-of-sight channels. Intelligent reflecting surface (IRS) is capable of reconfiguring the propagation environment, and thus is an attractive solution for integrating with UAV to facilitate the security in wireless networks. In this paper, we investigate the secure transmission design for an IRSassisted UAV network in the presence of an eavesdropper. With the aim at maximizing the average secrecy rate, the trajectory of UAV, the transmit beamforming, and the phase shift of IRS are jointly optimized. To address this sophisticated problem, we decompose it into three sub-problems and resort to an iterative algorithm to solve them alternately. First, we derive the closedform solution to the active beamforming. Then, with the optimal transmit beamforming, the passive beamforming optimization problem of fractional programming is transformed into corresponding parametric sub-problems. Moreover, the successive convex approximation is applied to deal with the non-convex UAV trajectory optimization problem by reformulating a convex problem which serves as a lower bound for the original one. Simulation results validate the effectiveness of the proposed scheme and the performance improvement achieved by the joint trajectory and beamforming design.
\end{abstract}

Index Terms-Intelligent reflecting surface, joint beamforming design, secure transmission, trajectory optimization, unmanned aerial vehicle.

\section{INTRODUCTION}

The advancement of unmanned aerial vehicles (UAVs) has motivated a plethora of applications of UAVs in military,

Manuscript received June 10, 2021; revised September 01, 2021 and November 04, 2021; accepted December 14, 2021. The work was supported by the National Key R\&D Program of China under Grant 2020YFB1807002, the National Natural Science Foundation of China (NSFC) under Grant 61871065, and the open research fund of State Key Laboratory of Integrated Services Networks under Grant ISN22-22. Part of this work is presented in WCSP'21 [1]. The associate editor coordinating the review of this paper and approving it for publication was J. Xu. (Corresponding author: Nan Zhao.)

$\mathrm{X}$. Pang and N. Zhao are with the Key Laboratory of Intelligent Control and Optimization for Industrial Equipment of Ministry of Education, Dalian University of Technology, Dalian 116024, P. R. China, and also with the State Key Laboratory of Integrated Services Networks, Xidian University, Xi'an, 710071, P. R. China (e-mail: xiaoweipang00@mail.dlut.edu.cn, zhaonan@dlut.edu.cn).

J. Tang is with the School of Electronic and Information Engineering, South China University of Technology, Guangzhou 510641, P.R. China (email: eejtang@scut.edu.cn).

$\mathrm{C}$. $\mathrm{Wu}$ is with the Graduate School of Informatics and Engineering, The University of Electro-Communications, Chofu 182-8585, Japan (e-mail: celimuge@uec.ac.jp).

D. Niyato is with the School of Computer Science and Engineering, Nanyang Technological University, Singapore 639798 (e-mail: dniyato@ntu.edu.sg).

K.-K. Wong is with the Department of Electronic and Electrical Engineering, University College London, London WC1E 6BT, U.K. (e-mail: kaikit.wong@ucl.ac.uk). civilian and commercial domains [2], including aerial inspection, cargo transport, search and rescue, video steaming, etc. Specifically, thanks to high maneuverability and cost-effective deployment, UAVs are gaining popularity in serving as aerial communication platforms to enhance the coverage, capacity and energy efficiency of existing wireless networks [3]-[5]. On the other hand, integrating UAVs into cellular networks as aerial users is envisioned to yield significant performance enhancement in network reliability and throughput [6]. In particular, UAVs are more likely to establish line-of-sight (LoS) wireless links [7], resulting in better air-ground communication quality.

Compared to the terrestrial communication infrastructure that is conventionally stationary, the three-dimensional (3D) mobility of UAVs offers a new design degree-of-freedom (DoF) to boost network performance. Deploying UAVs as quasistationary aerial base stations (BSs), Alzenad et al. proposed an efficient placement method for UAV-BSs to maximize the number of covered users with the minimum transmit power in [8]. Furthermore, they investigated a novel 3D UAV-BS placement to maximize the number of covered users with different quality of service requirements in [9]. Employing the UAV's mobility, Xu et al. solved the sum energy maximization problem for a UAV-enabled multiuser wireless power transfer system in [10] by optimizing the UAV's trajectory. In [11], the UAV trajectory was jointly optimized with power control and user scheduling and association by $\mathrm{Wu}$ et al., to maximize the minimum throughput among all users. In [12], the sum rate of all users served was maximized by jointly optimizing the UAV trajectory and the BS precoding. Furthermore, Shen et al. investigated a UAV-enabled interference channel in [13], where the joint trajectory and power control problem was resolved to maximize the aggregate sum rate. Different from the previous works that focused on the horizontal UAV trajectory, Yao et al. in [14] exploited the UAV's 3D maneuver together with the transmit power adaptation to maximize the average secrecy rate.

Despite the appealing advantages, UAV communication networks still face many new challenges. Compared to airair wireless links, air-ground channels are more prone to blockages caused by obstacles and mountainous terrain, which restrains the performance of UAV systems [15]. Another crucial issue is that the UAV broadcasting channels are more easily exposed to eavesdropping, which has spawned extensive research on the secure UAV transmission. To address this issue, employing jamming is envisioned as an effective solution to combat the wiretapping without degrading the legitimate transmission. Specifically, the trajectory, resource allocation 
and jamming policy were jointly designed in [16] and [17] to maximize the average minimum secrecy rate and the system energy efficiency, respectively. Furthermore, artificial jamming can be jointly optimized with the transmit beamforming at UAV to achieve secure transmission for all the legitimate users as studied by Chen et al. in [18]. It is worth noting that intelligent reflecting surface (IRS) is a promising solution to address these issues, due to its capability of restructuring wireless propagation environment [19]. Composed of a large array of reflecting elements, IRS can reflect the incident signal passively to either enhance the desired signal or suppress the interference with no need of any radio-frequency (RF) chains [20]. Motivated by this, there have been considerable works that utilize IRSs in terrestrial wireless networks to boost throughput [21], energy efficiency [22], as well as security [23].

Recently, IRS has attracted significant attention to be integrated into existing UAV networks to further unleash the air-ground network potential [24], [25]. There are mainly two paradigms for combining IRS and UAV to meet diverse network demands, depending on the installation of IRS. One type is the IRS-assisted UAV communication network [26][29], where the IRS installed on the surface of a building is introduced to help reflect the signal between the UAV and ground nodes. In [26], Li et al. jointly optimized the UAV trajectory and the IRS passive beamforming, intending to maximize the average achievable rate. In addition, Ge et al. employed multiple IRSs to assist UAV communications in [27], where the UAV trajectory, active beamforming and passive beamforming were jointly optimized to maximize the received power at the user. The joint design of UAV trajectory, IRS scheduling, and resource allocation was investigated in [28] by Wei et al. to maximize the system sum rate. Furthermore, a novel secure IRS-assisted UAV system was presented in [29], and the secrecy rate was maximized via the joint design of trajectory, transmit power and phase shifters. Another study case considers mounting the IRS on a UAV so as to enable the intelligent reflection from the sky [30][32]. Benefiting from the high mobility of UAV, the location of IRS is able to change accordingly, offering a new DoF for performance improvement. Deploying the IRS on UAV to achieve the energy-efficient end-to-end communication, Mohamed et al. jointly designed the beamforming vector at the BS and the phase shift matrix of the IRS in [30]. In [31], a reinforcement learning approach was adopted to model the propagation environment for effective deployment of a UAVcarried IRS. Due to the limited on-board energy of UAV, the IRS was anticipated to harvest energy from the unreflected fraction of signals to power itself. In addition, an IRS-UAV based multiple-input single-output (MISO) NOMA system was proposed in [32]. Therein, the location of IRS-UAV was optimized first, followed by optimizing the transmit beamforming and the phase shift of IRS alternately. Nevertheless, these works only studied the placement of static UAVs while the 3D mobility of UAVs has not been utilized.

Most of the existing works employ the IRS to improve the sum rate, while few of them consider dealing with the threat of eavesdropping. When the legitimate and eavesdropping users are in the same direction, conventional beamforming and artificial noise cannot fully ensure the secure transmission. Motivated by this, in this paper, we utilize an IRS to enhance the quality of the legitimate transmission while degrading that of the eavesdropping, enabling a secure transmission scheme for the IRS-assisted UAV network. Unlike the existing optimization designs for IRS-assisted UAV networks that either consider single-antenna UAVs or transmission without potential eavesdropping, our goal is to maximize the average achievable secrecy rate by jointly optimizing the transmit beamforming and the trajectory for the UAV and the passive beamforming for the IRS. The main contributions of this paper are summarized as follows.

- We present an IRS-assisted UAV network, where the IRS is employed to help the UAV transmit confidential information to the ground user in the presence of an eavesdropper. To guarantee the secure communication between the UAV and the legitimate user, the UAV trajectory, the transmit beamforming, and the phase shift of IRS are jointly optimized to maximize the average achievable secrecy rate during the flight.

- The original problem is intractable and hence is decomposed into three sub-problems. An iterative algorithm is developed to solve the sub-problems in an alternating manner until convergence. Given the other two variables, the closed-form solution to the transmit beamforming at the UAV is easy to acquire. Due to the induction of IRS and the eavesdropper, the optimization problems of IRS passive beamforming and UAV trajectory are complicated and non-convex.

- To obtain the phase shift matrix of IRS, a fractional programming method is adopted by recasting the problem into a series of parametric sub-problems. Furthermore, a closed-form solution is obtained by substituting the objective with its upper bound. Utilizing the successive convex approximation (SCA), the non-convex optimization problem of UAV trajectory is converted into a convex one and solved iteratively.

The remainder of this paper is structured as follows. In Section II, we introduce the system model and formulate the joint optimization problem. Furthermore, the problem is divided into three sub-problems and solved by an iterative algorithm in Section III. Section IV presents the simulation results to demonstrate the performance of the proposed design. Finally, we conclude the work in Section V.

Notation: Vectors and matrices are denoted by $\mathbf{a}$ and $\mathbf{A}$, respectively. $(\cdot)^{T},(\cdot)^{*}$ and $(\cdot)^{H}$ refer to the transpose, the conjugate, and the Hermitian operations, respectively. $\|\cdot\|$ is the Euclidean norm of a vector and $|\cdot|$ represents the absolute value of a complex scalar. $\otimes$ denotes the Kronecker product. For a complex number $a, \arg (a)$ and $\operatorname{Re}(a)$ return its phase and real part, respectively. $\lambda_{\max }(\mathbf{A})$ returns the maximum eigenvalue of $\mathbf{A} \cdot \operatorname{diag}(\mathbf{a})$ stands for a diagonal matrix whose diagonal elements stem from $\mathbf{a}$.

\section{System Model And Problem Formulation}

In this section, we first present the system and channel models. Then, the joint optimization problem is formulated 


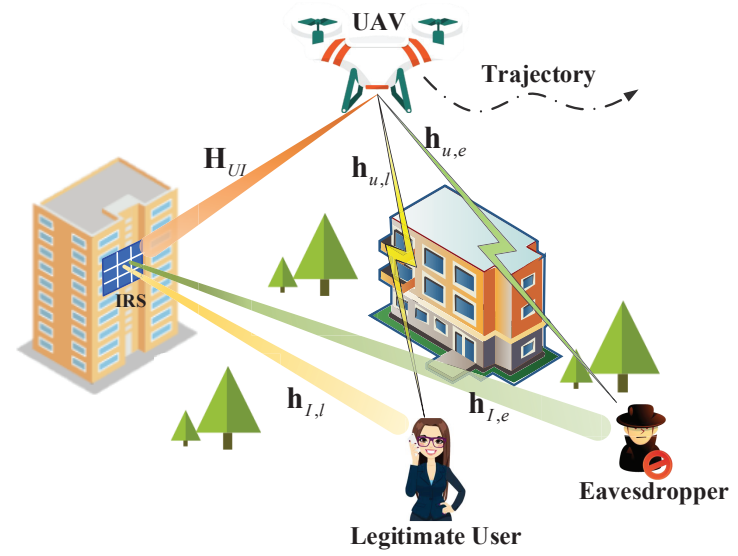

Fig. 1. Secure transmission in an IRS-assisted UAV wireless network.

to maximize the average secrecy rate.

\section{A. System Model}

We consider an IRS-assisted UAV wireless network exposed to the eavesdropping, as illustrated in Fig. 1. Assume that the eavesdropper is an active user but is untrusted and has no authority to access the confidential information between the UAV and the legitimate user. In particular, the direct link between the UAV and the ground user is severely blocked by ground obstacles due to the complicated urban environment. To enhance the legitimate transmission while combating the eavesdropping, an IRS is mounted on a building facade to assist the air-ground communication by reconfiguring the propagation environment. Assume that the UAV is equipped with $N_{u}$ antennas, while both the user and the eavesdropper have a single antenna. The UAV is assumed to fly at a fixed altitude $H_{u}$ above the ground within a time period $T$. For ease of tractability, we divide $T$ into $N$ small time slots so that the location of UAV can be approximately considered unchanged in each time slot.

Without loss of generality, a 3D Cartesian coordinate system is considered, where the horizontal coordinates of the legitimate user and the eavesdropper are denoted by $\mathbf{C}_{l}=\left[x_{l}, y_{l}\right]^{T}$ and $\mathbf{C}_{e}=\left[x_{e}, y_{e}\right]^{T}$, respectively. The first element of IRS is regarded as the reference point which is assumed to be located at the horizontal location $\mathbf{C}_{I}=\left[x_{I}, y_{I}\right]^{T}$ with the height $H_{I}$. The location of UAV projected onto the ground in the $n$th time slot is denoted as $\mathbf{q}[n]=[x[n], y[n]]^{T}, n=1,2, \ldots, N$. The initial and final locations of UAV during the flight are given as $\mathbf{q}_{I}$ and $\mathbf{q}_{F}$, respectively. As a consequence, the following mobility constraints of UAV should be satisfied.

$$
\begin{aligned}
& \mathbf{q}[1]=\mathbf{q}_{I}, \quad\left\|\mathbf{q}[N]-\mathbf{q}_{F}\right\|^{2} \leq\left(\frac{V_{m} T}{N}\right)^{2}, \\
& \|\mathbf{q}[n+1]-\mathbf{q}[n]\|^{2} \leq\left(\frac{V_{m} T}{N}\right)^{2}, n=1, \ldots, \mathcal{N}-1,
\end{aligned}
$$

where $V_{m}$ is the maximum speed of UAV and $\left(V_{m} T\right) / N$ denotes the maximum horizontal distance that the UAV can fly in each time slot.
Assume that the IRS consists of a uniform planar array (UPA) with $M=M_{x} M_{z}$ reflecting elements, where $M_{x}$ and $M_{z}$ denote the number of elements along the $x$-axis and $z$ axis, respectively. The phase shift matrix is denoted as $\boldsymbol{\Phi}[n]=$ $\operatorname{diag}\left(e^{j \varphi_{1}[n]}, \ldots, e^{j \varphi_{m}[n]}, \ldots, e^{j \varphi_{M}[n]}\right)$, where $\varphi_{m}[n]$ represents the phase shift incurred by the $m$ th reflecting element in the $n$th time slot.

\section{B. Channel Model}

Due to the obstacles such as buildings and trees in the complex environment, the LoS link from the UAV to the ground nodes are blocked and the direct channels consist of lots of scattering components. As a result, the UAV-ground channels follow Rayleigh block fading ${ }^{1}$, with the channel model from the UAV to the legitimate user and that to the eavesdropper given respectively as

$$
\begin{aligned}
\mathbf{h}_{u, l}^{H}[n] & =\sqrt{\rho d_{u, l}^{-\alpha}[n]} \widetilde{\mathbf{h}}_{u, l}[n] \in \mathbb{C}^{1 \times N_{u},} \\
\mathbf{h}_{u, e}^{H}[n] & =\sqrt{\rho d_{u, e}^{-\alpha}[n]} \widetilde{\mathbf{h}}_{u, e}[n] \in \mathbb{C}^{1 \times N_{u}},
\end{aligned}
$$

where $\rho$ represents the channel power gain at the reference distance $d_{0}=1 \mathrm{~m}, \alpha$ is the path-loss exponent, $d_{u, i}[n]=$ $\sqrt{\left\|\mathbf{q}[n]-\mathbf{C}_{i}\right\|^{2}+H_{u}^{2}}$ denotes the distance from the UAV to the secure user or the eavesdropper in the $n$th time slot, $i \in$ $\{l, e\}$. The elements of $\widetilde{\mathbf{h}}_{u, i}[n](i \in\{l, e\})$ are assumed to be independent and identically distributed complex Gaussian variable with zero mean and unit variance.

Similar to the assumptions in [27] and [33], the channel between the UAV and IRS is considered to be dominated by LoS links thanks to the vertical heights of UAV and IRS. Furthermore, Doppler effect caused by the UAV mobility is supposed to be well compensated. Thus, the UAV-IRS link can be modeled as

$$
\mathbf{H}_{U I}[n]=\sqrt{\rho d_{U I}^{-2}[n]} \mathbf{a}_{M}^{T}[n] \mathbf{a}_{N_{U}}[n] \in \mathbb{C}^{M \times N_{u}},
$$

where $d_{U I}[n]=\sqrt{\left\|\mathbf{q}[n]-\mathbf{C}_{I}\right\|^{2}+\left(H_{u}-H_{I}\right)^{2}}$ denotes the 3D distance from the UAV to the IRS in the $n$th time slot. Particularly, $\mathbf{a}_{M}^{T}[n]$ and $\mathbf{a}_{N_{u}}[n]$ are array responses in the $n$th time slot, which are obtained in the following.

With the element spacing of IRS denoted by $\widetilde{d}_{x}$ and $\widetilde{d}_{z}$ in Axis $x$ and Axis $z$, respectively, the receive array response of IRS can be expressed as

$$
\mathbf{a}_{M}[n]=\mathbf{a}_{x}(\phi[n], \theta[n]) \otimes \mathbf{a}_{z}(\phi[n], \theta[n]),
$$

where

$$
\begin{aligned}
& \mathbf{a}_{x}(\phi[n], \theta[n])=\left[1, \ldots, e^{-j \frac{2 \pi}{\lambda} \widetilde{d}_{x}\left(M_{x}-1\right) \sin \theta[n] \cos \phi[n]}\right], \\
& \mathbf{a}_{z}(\phi[n], \theta[n])=\left[1, \ldots, e^{-j \frac{2 \pi}{\lambda} \widetilde{d}_{z}\left(M_{z}-1\right) \cos \theta[n]}\right], \\
& \sin \theta[n] \cos \phi[n]=\frac{x_{I}-x[n]}{d_{U I}[n]}, \cos \theta[n]=\frac{H_{u}-H_{I}}{d_{U I}[n]} .
\end{aligned}
$$

$\phi[n]$ denotes the azimuth angle of arrival (AoA) and $\theta[n]$ represents the elevation AoA in the $n$th time slot. $\lambda$ is the

\footnotetext{
${ }^{1}$ Although we cannot obtain the accurate air-ground CSI in advance, it can provide a performance upper bound for more practical designs with statistical or partial CSI only.
} 
carrier wavelength.

The antennas equipped at the UAV is assumed to be a uniform linear array (ULA). With the known angle-of-departure (AoD) $\phi_{D}[n]$ and the antenna separation $\widetilde{d}$, the transmit array response can be expressed as

$$
\mathbf{a}_{N_{U}}[n]=\left[1, e^{-j \frac{2 \pi}{\lambda} \widetilde{d} \cos \phi_{D}[n]}, \ldots, e^{-j \frac{2 \pi}{\lambda} \widetilde{d}\left(N_{u}-1\right) \cos \phi_{D}[n]}\right] .
$$

Compared with the UAV-IRS channel, the IRS-ground channels are much more complicated and include both LoS and NLoS components. We model the channel from the IRS to a ground node $i$ as a Rician block fading model, $i \in\{l, e\}$, which can be given as

$$
\mathbf{h}_{I, i}[n]=\sqrt{\rho d_{I, i}^{-\beta}}\left(\sqrt{\frac{\mathcal{K}}{\mathcal{K}+1}} \overline{\mathbf{h}}_{I, i}^{L}[n]+\sqrt{\frac{1}{\mathcal{K}+1}} \widetilde{\mathbf{h}}_{I, i}^{N}[n]\right),
$$

where $d_{I, i}$ represents the distance between the IRS and the ground node, $\beta$ is the path-loss exponent, and $\mathcal{K}$ denotes the Rician factor. With the azimuth and elevation AoDs for the LoS link from the IRS to node $i$ denoted by $\phi_{i}$ and $\theta_{i}$, the LoS component can be obtained as

$$
\begin{gathered}
\overline{\mathbf{h}}_{I, i}^{L}[n]=\left[1, \ldots, e^{\left.-j \frac{2 \pi}{\lambda} \widetilde{d}_{x}\left(M_{x}-1\right) \sin \theta_{i} \cos \phi_{i}\right] \otimes}\right. \\
{\left[1, \ldots, e^{-j \frac{2 \pi}{\lambda} \widetilde{d}_{z}\left(M_{z}-1\right) \cos \theta_{i}}\right],} \\
\sin \theta_{i} \cos \phi_{i}=\frac{x_{i}-x_{I}}{d_{I, i}}, \quad \cos \theta_{i}=\frac{H_{I}}{d_{I, i}} .
\end{gathered}
$$

In addition, the NLoS component $\widetilde{\mathbf{h}}_{I, i}^{N}[n] \in \mathbb{C}^{M \times 1}$ is modeled as complex Gaussian distributed with zero mean and unit variance, i.e., $\widetilde{\mathbf{h}}_{I, i}^{N}[n] \sim \mathcal{C N}(\mathbf{0}, \mathbf{I})$. Based on the existing channel estimation approaches for IRS-assisted communications [34], we assume that all the channel state information (CSI) is available in this paper.

The received signal at the legitimate user and at the eavesdropper in the $n$th time slot can be respectively modeled as

$$
\begin{aligned}
& y_{l}[n]=\left(\mathbf{h}_{u, l}^{H}[n]+\mathbf{h}_{I, l}[n] \boldsymbol{\Phi}[n] \mathbf{H}_{U I}[n]\right) \mathbf{w}[n] x[n]+z_{l}[n], \\
& y_{e}[n]=\left(\mathbf{h}_{u, e}^{H}[n]+\mathbf{h}_{I, e}[n] \boldsymbol{\Phi}[n] \mathbf{H}_{U I}[n]\right) \mathbf{w}[n] x[n]+z_{e}[n],
\end{aligned}
$$

where $x[n]$ is the transmitted signal, $\mathbf{w}[n] \in \mathbb{C}^{N_{u} \times 1}$ denotes the beamforming vector at the UAV, and $z_{l}[n] \sim \mathcal{C N}\left(0, \sigma_{l}^{2}\right)$ and $z_{e}[n] \sim \mathcal{C N}\left(0, \sigma_{e}^{2}\right)$ denote the additive white Gaussian noise (AWGN) at the secure user and the eavesdropper, respectively.

\section{Problem Formulation}

Based on the system and channel models, the achievable rate of the legitimate user and that of the eavesdropper in the $n$th time slot can be respectively given as

$$
\begin{aligned}
& R_{L}[n]=\log _{2}\left(1+\frac{\left|\left(\mathbf{h}_{u, l}^{H}[n]+\mathbf{h}_{I, l}^{H}[n] \mathbf{\Phi}[n] \mathbf{H}_{U I}[n]\right) \mathbf{w}[n]\right|^{2}}{\sigma_{l}^{2}}\right), \\
& R_{E}[n]=\log _{2}\left(1+\frac{\left|\left(\mathbf{h}_{u, e}^{H}[n]+\mathbf{h}_{I, e}^{H}[n] \mathbf{\Phi}[n] \mathbf{H}_{U I}[n]\right) \mathbf{w}[n]\right|^{2}}{\sigma_{e}^{2}}\right) .
\end{aligned}
$$

Furthermore, the average achievable secrecy rate between the UAV and the legitimate user can be calculated as

$$
\bar{R}_{s}=\frac{1}{N} \sum_{n=1}^{N}\left[R_{L}[n]-R_{E}[n]\right]^{+},
$$

where $[x]^{+} \triangleq \max (x, 0)$. Note that if the value of $R_{L}[n]-$ $R_{E}[n]$ is negative in the $n$th time slot, we can make the transmit power $P_{u}=0$, resulting in $R_{s}[n]=R_{L}[n]-R_{E}[n]=0$. Thus, the secrecy rate can be always guaranteed to be nonnegative via adjusting the transmit power. In this regard, the operator $[.]^{+}$can be removed without changing the value of (14).

To ensure the secure transmission, our goal is to maximize the average achievable secrecy rate by jointly optimizing the UAV trajectory $\mathbf{Q} \triangleq\{\mathbf{q}[n], \forall n\}$, the transmit beamforming $\mathbf{W} \triangleq\{\mathbf{w}[n], \forall n\}$, and the phase shift matrix $\mathbf{\Psi} \triangleq\{\boldsymbol{\Phi}[n], \forall n\}$. Accordingly, the joint optimization problem can be formulated as

$$
\begin{aligned}
\max _{\mathbf{Q}, \mathbf{W}, \mathbf{\Psi}} & \bar{R}_{s} \\
\text { s.t. } & \mathbf{q}[1]=\mathbf{q}_{I},\left\|\mathbf{q}[N]-\mathbf{q}_{F}\right\|^{2} \leq\left(\frac{V_{m} T}{N}\right)^{2}, \\
& \|\mathbf{q}[n+1]-\mathbf{q}[n]\|^{2} \leq\left(\frac{V_{m} T}{N}\right)^{2}, n=1, \ldots, N-1, \\
& 0 \leq \varphi_{m}[n] \leq 2 \pi, \forall m, \forall n, \\
& \|\mathbf{w}[n]\|^{2} \leq P_{u}, \forall n,
\end{aligned}
$$

where $P_{u}$ is the maximum transmit power of UAV. It is difficult to handle this problem since the optimization variables are coupled in the objective function. To address this issue, the joint problem will be decomposed into three sub-problems and solved iteratively in the next section.

\section{JOINT OPTIMIZATION FOR SECRECy RATE MAXIMIZATION}

In this section, an iterative algorithm is proposed by optimizing the three variables in an alternating manner to achieve a high-quality suboptimal solution to the secrecy rate maximization problem. Specifically, the optimal solution to the active beamforming can be obtained directly in a closed form. For the non-convex optimization problems of phase shift and trajectory, we resort to SCA and solve their approximated problems instead.

\section{A. Active Beamforming Design for $U A V$}

By fixing $\mathbf{Q}$ and $\boldsymbol{\Psi}$, the transmit beamforming sub-problem can be equivalently reformulated as

$$
\begin{array}{ll}
\max _{\mathbf{w}[n]} \frac{\left|\widetilde{\mathbf{h}}_{l}[n] \mathbf{w}[n]\right|^{2}+\sigma_{l}^{2}}{\left|\widetilde{\mathbf{h}}_{e}[n] \mathbf{w}[n]\right|^{2}+\sigma_{e}^{2}} \\
\text { s.t. } & \|\mathbf{w}[n]\|^{2} \leq P_{u}, \forall n,
\end{array}
$$

where the concatenated channels are denoted by $\widetilde{\mathbf{h}}_{l}[n]=$ $\mathbf{h}_{u, l}^{H}[n]+\mathbf{h}_{I, l}^{H}[n] \boldsymbol{\Phi}[n] \mathbf{H}_{U I}[n]$ and $\widetilde{\mathbf{h}}_{e}[n]=\mathbf{h}_{u, e}^{H}[n]+$ 
$\mathbf{h}_{I, e}^{H}[n] \boldsymbol{\Phi}[n] \mathbf{H}_{U I}[n]$. By defining $\mathbf{H}_{l}[n]=\widetilde{\mathbf{h}}_{l}^{H}[n] \widetilde{\mathbf{h}}_{l}[n]$ and $\mathbf{H}_{e}[n]=\widetilde{\mathbf{h}}_{e}^{H}[n] \widetilde{\mathbf{h}}_{e}[n]$, (16) can be rewritten as

$$
\begin{aligned}
\max _{\mathbf{w}[n]} & \frac{\mathbf{w}^{H}[n] \mathbf{H}_{l}[n] \mathbf{w}[n]+\sigma_{l}^{2}}{\mathbf{w}^{H}[n] \mathbf{H}_{e}[n] \mathbf{w}[n]+\sigma_{e}^{2}} \\
\text { s.t. } & \mathbf{w}^{H}[n] \mathbf{w}[n] \leq P_{u}, \forall n .
\end{aligned}
$$

According to [35], the optimal solution to the transmit beamforming is given by $\mathbf{w}_{\text {opt }}[n]=\sqrt{P_{u}} \mathbf{v}_{\max }[n]$, where $\mathbf{v}_{\max }[n]$ is the eigenvector corresponding to the largest eigenvalue of the $\operatorname{matrix}\left(\mathbf{H}_{e}[n] P_{u}+\sigma_{e}^{2} \mathbf{I}_{N_{u}}\right)^{-1}\left(\mathbf{H}_{l}[n] P_{u}+\sigma_{l}^{2} \mathbf{I}_{N_{u}}\right) . \mathbf{I}_{N_{u}} \in$ $\mathbb{C}^{N_{u} \times N_{u}}$ represents an identity matrix.

\section{B. Passive Beamforming Design for IRS}

We redefine $\boldsymbol{\Phi}[n]=\operatorname{diag}\left(\theta_{1}[n], \ldots, \theta_{M}[n]\right)$, which has unit modulus constraints. With the optimized active beamforming and given UAV trajectory, the optimal phase shifts of IRS can be obtained by solving

$$
\begin{aligned}
\max _{\mathbf{\Phi}[n]} & \frac{\left|\left(\mathbf{h}_{u, l}^{H}[n]+\mathbf{h}_{I, l}^{H}[n] \mathbf{\Phi}[n] \mathbf{H}_{U I}[n]\right) \mathbf{w}[n]\right|^{2}+\sigma_{l}^{2}}{\left|\left(\mathbf{h}_{u, e}^{H}[n]+\mathbf{h}_{I, e}^{H}[n] \mathbf{\Phi}[n] \mathbf{H}_{U I}[n]\right) \mathbf{w}[n]\right|^{2}+\sigma_{e}^{2}} \\
\text { s.t. } & \left|\theta_{m}[n]\right|=1, m=1, \ldots, M .
\end{aligned}
$$

First, introduce an auxiliary vector $\mathbf{u}^{H}[n]=\left[\theta_{1}[n], \ldots, \theta_{M}[n]\right]$. Due to the equality $\mathbf{a}^{H} \boldsymbol{\Phi}[n] \mathbf{b}=\mathbf{u}^{H}[n] \operatorname{diag}\left\{\mathbf{a}^{H}\right\} \mathbf{b}$, we have $\mathbf{h}_{I, l}^{H}[n] \boldsymbol{\Phi}[n] \mathbf{H}_{U I}[n] \mathbf{w}[n]=\mathbf{u}^{H}[n] \operatorname{diag}\left\{\mathbf{h}_{I, l}^{H}[n]\right\} \mathbf{H}_{U I}[n] \mathbf{w}[n]$. Based on this, the problem (18) can be rewritten as

$$
\begin{aligned}
\max _{\mathbf{u}[n]} & \frac{\left|\mathbf{a}_{l}[n]+\mathbf{u}^{H}[n] \mathbf{b}_{l}[n]\right|^{2}+\sigma_{l}^{2}}{\left|\mathbf{a}_{e}[n]+\mathbf{u}^{H}[n] \mathbf{b}_{e}[n]\right|^{2}+\sigma_{e}^{2}} \\
\text { s.t. } & \left|\theta_{m}[n]\right|=1, m=1, \ldots, M,
\end{aligned}
$$

where $\mathbf{a}_{l}[n]=\mathbf{h}_{u, l}^{H}[n] \mathbf{w}[n], \mathbf{b}_{l}[n]=\operatorname{diag}\left\{\mathbf{h}_{I, l}^{H}[n]\right\} \mathbf{H}_{U I}[n] \mathbf{w}[n]$, $\mathbf{a}_{e}[n]=\mathbf{h}_{u, e}^{H}[n] \mathbf{w}[n]$, and $\mathbf{b}_{e}[n]=\operatorname{diag}\left\{\mathbf{h}_{I, e}^{H}[n]\right\} \mathbf{H}_{U I}[n] \mathbf{w}[n]$. To make it solvable, we further transform (19) into an equivalent form as

$$
\begin{aligned}
\min _{\mathbf{u}[n]} & \frac{\left|\mathbf{a}_{e}[n]+\mathbf{u}^{H}[n] \mathbf{b}_{e}[n]\right|^{2}+\sigma_{e}^{2}}{\left|\mathbf{a}_{l}[n]+\mathbf{u}^{H}[n] \mathbf{b}_{l}[n]\right|^{2}+\sigma_{l}^{2}} \\
\text { s.t. } & \left|\theta_{m}[n]\right|=1, m=1, \ldots, M,
\end{aligned}
$$

which belongs to fractional programming and can be converted into a series of parametric sub-problems according to [36]. In the $r$ th iteration, we proceed to solve the following subproblem.

$$
\begin{array}{ll}
\min _{\mathbf{u}[n]} & \left|\mathbf{a}_{e}[n]+\mathbf{u}^{H}[n] \mathbf{b}_{e}[n]\right|^{2}+\sigma_{e}^{2} \\
& -\eta^{(r-1)}\left(\left|\mathbf{a}_{l}[n]+\mathbf{u}^{H}[n] \mathbf{b}_{l}[n]\right|^{2}+\sigma_{l}^{2}\right) \\
\text { s.t. } & \left|\theta_{m}[n]\right|=1, m=1, \ldots, M,
\end{array}
$$

where $\eta^{(r)}(r \geq 0)$ is a nonnegative parameter with an initial value $\eta^{(0)}=0$, and can be updated by

$$
\eta^{(r)}=\frac{\left|\mathbf{a}_{e}[n]+\mathbf{u}^{(r) H}[n] \mathbf{b}_{e}[n]\right|^{2}+\sigma_{e}^{2}}{\left|\mathbf{a}_{l}[n]+\mathbf{u}^{(r) H}[n] \mathbf{b}_{l}[n]\right|^{2}+\sigma_{l}^{2}} .
$$

$\mathbf{u}^{(r) H}[n]$ is the solution obtained in the $r$ th iteration. It is worth pointing out that the objective function in (21a) is still non-convex and difficult to handle. Thus, we substitute it with a tractable upper bound. Specifically, based on [37], the relationship is detailed as

$$
\begin{aligned}
& \left|\mathbf{a}_{e}[n]+\mathbf{u}^{H}[n] \mathbf{b}_{e}[n]\right|^{2}+\sigma_{e}^{2}-\eta\left(\left|\mathbf{a}_{l}[n]+\mathbf{u}^{H}[n] \mathbf{b}_{l}[n]\right|^{2}+\sigma_{l}^{2}\right) \\
& =\mathbf{u}^{H}[n]\left(\mathbf{b}_{e}[n] \mathbf{b}_{e}^{H}[n]-\mathbf{b}_{l}[n] \mathbf{b}_{l}^{H}[n]\right) \mathbf{u}[n]+\left|\mathbf{a}_{e}[n]\right|^{2}+\sigma_{e}^{2} \\
& -2 \operatorname{Re}\left\{\mathbf{u}^{H}[n]\left(\mathbf{a}_{l}^{*}[n] \mathbf{b}_{l}[n]-\mathbf{a}_{e}^{*}[n] \mathbf{b}_{e}[n]\right)\right\}-\eta\left|\mathbf{a}_{l}[n]\right|^{2}-\eta \sigma_{l}^{2} \\
& \leq \lambda_{\max }(\mathbf{A}[n])\|\mathbf{u}[n]\|^{2}-2 \operatorname{Re}\left\{\mathbf{u}^{H}[n] \mathbf{B}[n]\right\}+\mathbf{C}[n],
\end{aligned}
$$

where $\mathbf{A}[n], \mathbf{B}[n]$ and $\mathbf{C}[n]$ are given by

$$
\begin{aligned}
\mathbf{A}[n]= & \mathbf{b}_{e}[n] \mathbf{b}_{e}^{H}[n]-\eta \mathbf{b}_{l}[n] \mathbf{b}_{l}^{H}[n], \\
\mathbf{B}[n]= & \left.\lambda_{\max }(\mathbf{A}[n]) \mathbf{I}-\mathbf{A}[n]\right) \tilde{\mathbf{u}}[n]+\eta \mathbf{a}_{l}^{*}[n] \mathbf{b}_{l}[n]-\mathbf{a}_{e}^{*}[n] \mathbf{b}_{e}[n], \\
\mathbf{C}[n]= & \tilde{\mathbf{u}}^{H}[n]\left(\lambda_{\max }(\mathbf{A}[n]) \mathbf{I}-\mathbf{A}[n]\right) \tilde{\mathbf{u}}[n] \\
& +\left|\mathbf{a}_{e}[n]\right|^{2}+\sigma_{e}^{2}-\eta\left|\mathbf{a}_{l}[n]\right|^{2}-\eta \sigma_{l}^{2} .
\end{aligned}
$$

Note that $\tilde{\mathbf{u}}[n]$ denotes the optimal solution to $\mathbf{u}[n]$ in the previous iteration. Thus, the phase shift optimization problem can be simplified as

$$
\begin{array}{ll}
\min _{\mathbf{u}[n]} & \lambda_{\max }(\mathbf{A}[n])\|\mathbf{u}[n]\|^{2}-2 \operatorname{Re}\left\{\mathbf{u}^{H}[n] \mathbf{B}[n]\right\} \\
\text { s.t. } & \left|\theta_{m}[n]\right|=1, m=1, \ldots, M .
\end{array}
$$

The unit modulus constraint $\left|\theta_{m}[n]\right|=1$ results in $\|\mathbf{u}[n]\|^{2}=$ $M$, which means that $\lambda_{\max }(\mathbf{A}[n])\|\mathbf{u}[n]\|^{2}$ is a constant. In this case, it is obvious that the objective function is minimized when $\operatorname{Re}\left\{\mathbf{u}^{H}[n] \mathbf{B}[n]\right\}$ is maximized. To achieve this, the phase of $\theta_{m}[n]$ is required to align to the phase of the $m$ th entry of $\mathbf{B}[n]$ which is recorded as $\mathbf{B}_{m}[n]$. Accordingly, the optimal solution to $\theta_{m}[n]$ is calculated as $\theta_{m}^{*}[n]=e^{j \arg \left(\mathbf{B}_{m}[n]\right)}$, and the optimal phase shift vector can be obtained as

$$
\mathbf{u}_{o p t}^{H}[n]=\left[e^{j \arg \left(\mathbf{B}_{1}[n]\right)}, \ldots, e^{j \arg \left(\mathbf{B}_{M}[n]\right)}\right] .
$$

Therefore, we have a closed-form solution in each iteration, which significantly reduces the computational complexity.

\section{Optimizing UAV Trajectory with Given Beamforming}

With the transmit beamforming and the phase shift matrix obtained by solving the abovementioned subproblems, the UAV trajectory optimization sub-problem can be separated from (15) as

$$
\begin{aligned}
\max _{\mathbf{Q}} & \frac{1}{N} \sum_{n=1}^{N}\left(R_{L}[n]-R_{E}[n]\right) \\
\text { s.t. } & \mathbf{q}[1]=\mathbf{q}_{I},\left\|\mathbf{q}[N]-\mathbf{q}_{F}\right\|^{2} \leq\left(\frac{V_{m} T}{N}\right)^{2}, \\
& \|\mathbf{q}[n+1]-\mathbf{q}[n]\|^{2} \leq\left(\frac{V_{m} T}{N}\right)^{2}, n=1, \ldots, N-1 .
\end{aligned}
$$

This problem is intractable due to the non-convex objective function, for which we can apply SCA to make it convex.

As observed, both $R_{L}[n]$ and $R_{E}[n]$ are neither convex nor concave. To deal with $R_{L}[n]$, we make mathematical operations on the received signal power at the legitimate user 
as

$$
\begin{aligned}
& \left|\left(\mathbf{h}_{u, l}^{H}[n]+\mathbf{h}_{I, l}^{H}[n] \mathbf{\Phi}[n] \mathbf{H}_{U I}[n]\right) \mathbf{w}[n]\right|^{2} \\
& =X_{l}[n] d_{u, l}^{-\alpha}[n]+Y_{l}[n] d_{u, l}^{-\frac{\alpha}{2}}[n] d_{U I}^{-1}[n]+Z_{l}[n] d_{U I}^{-2}[n],
\end{aligned}
$$

where the constant coefficients are derived as

$$
\begin{aligned}
& X_{l}[n]=\widetilde{\mathbf{h}}_{u, l}[n] \mathbf{W}[n] \widetilde{\mathbf{h}}_{u, l}^{H}[n], \\
& Y_{l}[n]=2 \operatorname{Re}\left\{\mathbf{G}_{l}[n] \mathbf{a}_{M}^{T}[n] \mathbf{a}_{N_{U}}[n] \mathbf{W}[n] \widetilde{\mathbf{h}}_{u, l}^{H}[n]\right\}, \\
& Z_{l}[n]=\mathbf{G}_{l}[n] \mathbf{a}_{M}^{T}[n] \mathbf{a}_{N_{U}}[n] \mathbf{W}[n] \mathbf{a}_{N_{U}}^{*}[n] \mathbf{a}_{N_{U}}^{H}[n] \mathbf{G}_{l}^{H}[n] .
\end{aligned}
$$

In (29), $\mathbf{W}[n]=\mathbf{w}[n] \mathbf{w}^{H}[n]$ and $\mathbf{G}_{l}[n]=\mathbf{h}_{I, l}^{H}[n] \boldsymbol{\Phi}[n]$.

Similarly, to make $R_{E}[n]$ solvable, the transformation on the signal power received at the eavesdropper can be given as

$$
\begin{aligned}
& \left|\left(\mathbf{h}_{u, e}^{H}[n]+\mathbf{h}_{I, e}^{H}[n] \boldsymbol{\Phi}[n] \mathbf{H}_{U I}[n]\right) \mathbf{w}[n]\right|^{2} \\
& =X_{e}[n] d_{u, e}^{-\alpha}[n]+Y_{e}[n] d_{u, e}^{-\frac{\alpha}{2}}[n] d_{U I}^{-1}[n]+Z_{e}[n] d_{U I}^{-2}[n] .
\end{aligned}
$$

where

$$
\begin{aligned}
& X_{e}[n]=\widetilde{\mathbf{h}}_{u, e}[n] \mathbf{W}[n] \widetilde{\mathbf{h}}_{u, e}^{H}[n], \\
& Y_{e}[n]=2 \operatorname{Re}\left\{\mathbf{G}_{e}[n] \mathbf{a}_{M}^{T}[n] \mathbf{a}_{N_{U}}[n] \mathbf{W}[n] \widetilde{\mathbf{h}}_{u, e}^{H}[n]\right\}, \\
& Z_{e}[n]=\mathbf{G}_{e}[n] \mathbf{a}_{M}^{T}[n] \mathbf{a}_{N_{U}}[n] \mathbf{W}[n] \mathbf{a}_{N_{U}}^{*}[n] \mathbf{a}_{N_{U}}^{H}[n] \mathbf{G}_{e}^{H}[n] .
\end{aligned}
$$

In (31b) and (31c), $\mathbf{G}_{e}[n]=\mathbf{h}_{I, e}^{H}[n] \boldsymbol{\Phi}[n]$.

By introducing auxiliary variables $\mathbf{t}_{1}=\left\{t_{1}[n], \forall n\right\}$ and $\mathbf{t}_{2}=\left\{t_{2}[n], \forall n\right\}$, the original problem (27) can be reformulated as

$$
\begin{aligned}
\max _{\substack{\mathbf{Q}_{1}, \mathbf{t}_{1}, \mathbf{t}_{2}, \mathbf{v}_{1}, \mathbf{v}_{2}, \mathbf{v}_{3}}} & \frac{1}{N} \sum_{n=1}^{N}\left(\log _{2}(e) t_{1}[n]-\log _{2}(e) t_{2}[n]\right) \\
\text { s.t. } & 1+\frac{\rho}{\sigma^{2}} D[n] \geq e^{t_{1}[n]} \\
& 1+\frac{\rho}{\sigma^{2}} E[n] \leq e^{t_{2}[n]}, \\
& \mathbf{q}[1]=\mathbf{q}_{I},\left\|\mathbf{q}[N]-\mathbf{q}_{F}\right\|^{2} \leq\left(\frac{V_{m} T}{N}\right)^{2}, \\
& \|\mathbf{q}[n+1]-\mathbf{q}[n]\|^{2} \leq\left(\frac{V_{m} T}{N}\right)^{2}, n=1, \ldots, N-1,
\end{aligned}
$$

where $D[n]$ and $E[n]$ can be expressed as

$$
\begin{aligned}
& D[n]=X_{l}[n] d_{u, l}^{-\alpha}[n]+Y_{l}[n] d_{u, l}^{-\frac{\alpha}{2}}[n] d_{U I}^{-1}[n]+Z_{l}[n] d_{U I}^{-2}[n], \\
& E[n]=X_{e}[n] d_{u, e}^{-\alpha}[n]+Y_{e}[n] d_{u, e}^{-\frac{\alpha}{2}}[n] d_{U I}^{-1}[n]+Z_{e}[n] d_{U I}^{-2}[n] .
\end{aligned}
$$

Note that the constraints (32b) and (32c) are still non-convex, wherein $D[n]$ and $E[n]$ should be converted into a concave one and a convex one, respectively. Motivated by this, we focus on finding approximate substitutions for $D[n]$ and $E[n]$ to convert the constraints into convex ones.

For convenience, we define several functions as

$$
\begin{aligned}
& F_{x}[n]=X_{l}[n] d_{u, l}^{-\alpha}[n], \\
& F_{y}[n]=Y_{l}[n] d_{u, l}^{-\frac{\alpha}{2}}[n] d_{U I}^{-1}[n], \\
& F_{z}[n]=Z_{l}[n] d_{U I}^{-2}[n] .
\end{aligned}
$$

Lemma 1: Non-concave $F_{x}[n]$ and $F_{y}[n]$ can be trans- formed into concave ones as

$$
\begin{aligned}
& F_{x}[n] \geq \mathcal{L}_{x}\left(\bar{v}_{1}[n], v_{1}[n]\right), \\
& F_{z}[n] \geq \mathcal{L}_{z}\left(\bar{v}_{3}[n], v_{3}[n]\right),
\end{aligned}
$$

where

$$
\begin{aligned}
& \mathcal{L}_{x}\left(\bar{v}_{1}[n], v_{1}[n]\right)=(1+\alpha) X_{l}[n] \bar{v}_{1}^{-\alpha}[n]-\alpha X_{l}[n] \bar{v}_{1}^{-\alpha-1}[n] v_{1}[n] \\
& \mathcal{L}_{z}\left(\bar{v}_{3}[n], v_{3}[n]\right)=3 Z_{l}[n] \bar{v}_{3}^{-2}[n]-2 Z_{l}[n] \bar{v}_{3}^{-3}[n] v_{3}[n]
\end{aligned}
$$

In addition, the following inequalities should be satisfied.

$$
\begin{aligned}
& d_{u, l}^{2}[n]+\bar{v}_{1}^{2}[n]-2 \bar{v}_{1}[n] v_{1}[n] \leq 0, \forall n, \\
& d_{U I}^{2}[n]+\bar{v}_{3}^{2}[n]-2 \bar{v}_{3}[n] v_{3}[n] \leq 0, \forall n .
\end{aligned}
$$

Proof: First, we introduce slack variables $\mathbf{v}_{1}=$ $\left\{v_{1}[n], \forall n\right\}$ and $\mathbf{v}_{3}=\left\{v_{3}[n], \forall n\right\}$, which satisfy

$$
d_{u, l}[n] \leq v_{1}[n], \quad d_{U I}[n] \leq v_{3}[n], \forall n .
$$

Recall the fact that a convex function is globally lower bounded by its first-order Taylor expansion at any local points. Since $v_{1}^{2}[n]$ is convex, with the given local point $\bar{v}_{1}[n]$, its firstorder Taylor expansion can be expressed as

$$
\begin{aligned}
v_{1}^{2}[n] & \geq \bar{v}_{1}^{2}[n]+2 \bar{v}_{1}[n]\left(v_{1}[n]-\bar{v}_{1}[n]\right) \\
& =-\bar{v}_{1}^{2}[n]+2 \bar{v}_{1}[n] v_{1}[n] .
\end{aligned}
$$

According to (40), we have $d_{u, l}[n]^{2}-v_{1}[n]^{2} \leq 0$. Therefore, by substituting $v_{1}[n]^{2}$ with its first-order Taylor approximation, (39a) can be obtained. Based on the same idea, (39b) can be easily proved.

Due to the fact that $\mathbf{W}[n]=\mathbf{w}[n] \mathbf{w}^{H}[n]$ is generally a positive semi-definite matrix which holds $\mathbf{W}[n] \succeq 0$, we have $X_{l}[n] \geq 0$ and $Z_{l}[n] \geq 0$ according to the expressions (29a) and (29c). Replace $d_{u, l}[n]$ and $d_{U I}[n]$ with $v_{1}[n]$ and $v_{3}[n]$, respectively, we have

$$
\begin{aligned}
& F_{x}[n] \geq X_{l}[n] v_{1}^{-\alpha}[n], \\
& F_{z}[n] \geq Z_{l}[n] v_{3}^{-2}[n],
\end{aligned}
$$

which are convex with $v_{1}[n]$ and $v_{3}[n]$. Hence, $F_{x}[n]$ and $F_{z}[n]$ are lower bounded by the corresponding first-order Taylor series of the right-side terms as

$$
\begin{aligned}
F_{x}[n] & \geq(1+\alpha) X_{l}[n] \bar{v}_{1}^{-\alpha}[n]-\alpha X_{l}[n] \bar{v}_{1}^{-\alpha-1}[n] v_{1}[n] \\
& \triangleq \mathcal{L}_{x}\left(\bar{v}_{1}[n], v_{1}[n]\right), \\
F_{z}[n] & \geq 3 Z_{l}[n] \bar{v}_{3}^{-2}[n]-2 Z_{l}[n] \bar{v}_{3}^{-3}[n] v_{3}[n] \\
& \triangleq \mathcal{L}_{z}\left(\bar{v}_{3}[n], v_{3}[n]\right),
\end{aligned}
$$

which are affine functions and also concave.

Nevertheless, $Y_{l}[n]$ is not necessarily positive or negative. When $Y_{l}[n] \geq 0$, through utilizing slack variables $v_{1}[n]$ and $v_{3}[n], F_{y}[n]$ can be approximated by the first-order Taylor series as

$$
\begin{aligned}
& F_{y}[n] \geq\left(2+\frac{\alpha}{2}\right) Y_{l}[n] \bar{v}_{1}^{-\frac{\alpha}{2}}[n] \bar{v}_{3}^{-1}[n] \\
& -\frac{\alpha}{2} Y_{l}[n] \bar{v}_{3}^{-1}[n] \bar{v}_{1}^{-\frac{\alpha}{2}-1}[n] v_{1}[n]-Y_{l}[n] \bar{v}_{1}^{-\frac{\alpha}{2}}[n] \bar{v}_{3}^{-2}[n] v_{3}[n] \\
& \triangleq \mathcal{L}_{y}\left(\bar{v}_{1}[n], \bar{v}_{3}[n], v_{1}[n], v_{3}[n]\right) .
\end{aligned}
$$

In contrast, if $Y_{l}[n]<0$, the above operations do not make 
sense anymore. Therefore, Lemma 2 is proposed to render $F_{y}[n]$ concave under this condition.

Lemma 2: When $Y_{l}[n]<0$, the concave lower bound of $F_{y}[n]$ can be given as

$$
F_{y}[n] \geq \mathcal{T}_{y}\left(\bar{V}_{1}[n], \bar{V}_{3}[n], V_{1}[n], V_{3}[n]\right),
$$

where

$$
\begin{aligned}
\mathcal{T}_{y}= & \frac{Y_{l}[n]}{2}\left[\left(V_{1}^{-\frac{\alpha}{2}}[n]+V_{3}^{-1}[n]\right)^{2}-(1+\alpha) \bar{V}_{1}^{-\alpha}[n]\right. \\
& \left.+\alpha \bar{V}_{1}^{-\alpha-1}[n] V_{1}[n]-3 \bar{V}_{3}^{-2}[n]+2 \bar{V}_{3}^{-3}[n] V_{3}[n]\right] .
\end{aligned}
$$

The inequality above is based on the following constraints:

$$
\begin{gathered}
V_{1}^{2}[n]+\left\|\mathbf{q}^{r}[n]\right\|^{2}-2\left(\mathbf{q}^{r}[n]-\mathbf{C}_{l}\right)^{T} \mathbf{q}[n]-\left\|\mathbf{C}_{l}\right\|^{2}+H_{u}^{2} \leq 0, \\
V_{3}^{2}[n]+\left\|\mathbf{q}^{r}[n]\right\|^{2}-2\left(\mathbf{q}^{r}[n]-\mathbf{C}_{I}\right)^{T} \mathbf{q}[n]-\left\|\mathbf{C}_{I}\right\|^{2}+H_{u I}^{2} \leq 0,
\end{gathered}
$$

where $H_{u I}=H_{u}-H_{I}$.

Proof: Particularly, we introduce another two relaxation variables $\mathbf{V}_{1}=\left\{V_{1}[n], \forall n\right\}$ and $\mathbf{V}_{3}=\left\{V_{3}[n], \forall n\right\}$, which satisfy

$$
V_{1}[n] \leq d_{u, l}[n], \quad V_{3}[n] \leq d_{U I}[n], \forall n .
$$

In this case, $Y_{l}[n] V_{1}^{-\frac{\alpha}{2}}[n] V_{3}^{-1}[n]$ serves as a lower bound for $F_{y}[n]$. Then, with the given local points $\bar{V}_{1}[n]$ and $\bar{V}_{3}[n]$, the alteration of $F_{y}[n]$ can be given as

$$
\begin{aligned}
F_{y}[n] & \geq Y_{l}[n] V_{1}^{-\frac{\alpha}{2}}[n] V_{3}^{-1}[n] \\
& =\frac{Y_{l}[n]}{2}\left[\left(V_{1}^{-\frac{\alpha}{2}}[n]+V_{3}^{-1}[n]\right)^{2}-V_{1}^{-\alpha}-V_{3}^{-2}[n]\right] \\
& \stackrel{(a)}{\geq} \frac{Y_{l}[n]}{2}\left[\left(V_{1}^{-\frac{\alpha}{2}}[n]+V_{3}^{-1}[n]\right)^{2}-(1+\alpha) \bar{V}_{1}^{-\alpha}[n]\right. \\
& \left.+\alpha \bar{V}_{1}^{-\alpha-1}[n] V_{1}[n]-3 \bar{V}_{3}^{-2}[n]+2 \bar{V}_{3}^{-3}[n] V_{3}[n]\right] \\
& \triangleq \mathcal{T}_{y}\left(\bar{V}_{1}[n], \bar{V}_{3}[n], V_{1}[n], V_{3}[n]\right),
\end{aligned}
$$

where Step (a) is based on the fact that the terms $V_{1}^{-\alpha}[n]$ and $V_{3}^{-2}[n]$ are convex. $\mathcal{T}_{y}$ can be easily proved to be concave under the condition $F_{y}[n]<0$ by using its Hessian matrix.

According to (50), we have $V_{1}^{2}[n]-d_{u, l}^{2}[n] \leq 0$, where $-d_{u, l}^{2}[n]=-\left\|\mathbf{q}[n]-\mathbf{C}_{l}\right\|^{2}-H_{u}^{2}$. Since the negative normsquared function $-\left\|\mathbf{q}[n]-\mathbf{C}_{l}\right\|^{2}$ is concave with respect to the variable vector $\mathbf{q}[n]$, it has a convex upper bound given as

$$
\begin{aligned}
& -\left\|\mathbf{q}[n]-\mathbf{C}_{l}\right\|^{2} \stackrel{(b)}{\leq}-\left\|\mathbf{q}^{r}[n]-\mathbf{C}_{l}\right\|^{2}-2\left(\mathbf{q}^{r}[n]-\mathbf{C}_{l}\right)^{T}\left(\mathbf{q}[n]-\mathbf{q}^{r}[n]\right) \\
& =-\left\|\mathbf{q}^{r}[n]\right\|^{2}+2 \mathbf{C}_{l}^{T} \mathbf{q}^{r}[n]-\left\|\mathbf{C}_{l}\right\|^{2}-2\left(\mathbf{q}^{r}[n]-\mathbf{C}_{l}\right)^{T}\left(\mathbf{q}[n]-\mathbf{q}^{r}[n]\right) \\
& =\left\|\mathbf{q}^{r}[n]\right\|^{2}-2\left(\mathbf{q}^{r}[n]-\mathbf{C}_{l}\right)^{T} \mathbf{q}[n]-\left\|\mathbf{C}_{l}\right\|^{2},
\end{aligned}
$$

where Step (b) follows the fact that the first-order Taylor approximation is a global upper estimator of a concave function. $\mathbf{q}^{r}[n]$ denotes the given point and $\mathbf{Q}^{r} \triangleq\left\{\mathbf{q}^{r}[n], \forall n\right\}$ denotes the trajectory of UAV in the $r$ th iteration. Obviously, (49a) can be obtained by substituting $-d_{u, l}^{2}[n]$ with its upper bound. Similarly, (49b) can be attained via applying the first-order Taylor expansion of $-d_{U I}^{2}[n]$.

Define a binary variable $I_{y}[n]$, which indicates $Y_{l}[n] \geq 0$ in the $n$th time slot if $I_{y}[n]=1$; otherwise, $I_{y}[n]=0$. Based on the above derivations, the concave lower bound for $D[n]$ can be obtained as

$$
\begin{aligned}
D[n] & =F_{x}[n]+F_{y}[n]+F_{z}[n] \\
& \stackrel{(c)}{\geq} \mathcal{L}_{x}[n]+\mathcal{L}_{z}[n]+I_{y}[n] \mathcal{L}_{y}[n]+\left(1-I_{y}[n]\right) \mathcal{T}_{y}[n] \\
& \triangleq \widetilde{D}[n] .
\end{aligned}
$$

In Step (c), we remove the parentheses for simplicity and replace the derived first-order Taylor approximations with $\mathcal{L}_{x}[n], \mathcal{L}_{z}[n], \mathcal{L}_{y}[n]$ and $\mathcal{T}_{y}[n]$.

In (32c), $e^{t_{2}[n]}$ is convex and hence is approximated by its first-order Taylor expansion at a given feasible point $\bar{t}_{2}[n]$. We have

$$
e^{t_{2}[n]} \geq e^{\bar{t}_{2}[n]}\left(t_{2}[n]-\bar{t}_{2}[n]+1\right) .
$$

As for $E[n]$ in the left side of (32c), it is required to be converted into a convex function. Similar to the operations on $D[n]$, new functions related to $E[n]$ are defined as

$$
\begin{aligned}
\mathcal{F}_{x}[n] & =X_{e}[n] d_{u, e}^{-\alpha}[n], \\
\mathcal{F}_{y}[n] & =Y_{e}[n] d_{u, e}^{-\frac{\alpha}{2}}[n] d_{U I}^{-1}[n], \\
\mathcal{F}_{z}[n] & =Z_{e}[n] d_{U I}^{-2}[n] .
\end{aligned}
$$

Contrary to $D[n]$ that needs a concave lower bound, $E[n]$ ought to be approximated by a convex upper bound. Motivated by Lemma 1, we introduce auxiliary variables $\mathbf{v}_{2}=$ $\left\{v_{2}[n], \forall n\right\}$ and $\mathbf{V}_{2}=\left\{V_{2}[n], \forall n\right\}$, which yield

$$
d_{u, e}[n] \leq v_{2}[n], \quad V_{2}[n] \leq d_{u, e}[n], \forall n .
$$

The conclusions are also tenable that $X_{e}[n] \geq 0$ and $Z_{e}[n] \geq$ 0 , and $Y_{e}[n]$ are not necessarily positive. To cope with $\mathcal{F}_{x}[n]$ and $\mathcal{F}_{z}[n]$, we utilize $V_{2}[n]$ and $V_{3}[n]$ to replace $d_{u, e}[n]$ and $d_{U I}[n]$, respectively, to obtain their upper approximations as

$$
\begin{aligned}
& \mathcal{F}_{x}[n] \leq X_{e}[n] V_{2}^{-\alpha}[n] \triangleq \mathcal{S}_{x}[n], \\
& \mathcal{F}_{z}[n] \leq Z_{e}[n] V_{3}^{-2}[n] \triangleq \mathcal{S}_{z}[n],
\end{aligned}
$$

which are convex. Moreover, when $Y_{e}[n] \geq 0, \mathcal{F}_{y}[n]$ is converted into a convex counterpart as

$$
\begin{aligned}
& \mathcal{F}_{y}[n] \leq \frac{Y_{e}[n]}{2}\left[\left(V_{2}^{-\frac{\alpha}{2}}[n]+V_{3}^{-1}[n]\right)^{2}-V_{2}^{-\alpha}-V_{3}^{-2}[n]\right] \\
& \leq \frac{Y_{e}[n]}{2}\left[\left(V_{2}^{-\frac{\alpha}{2}}[n]+V_{3}^{-1}[n]\right)^{2}-(1+\alpha) \bar{V}_{2}^{-\alpha}[n]\right. \\
& \left.+\alpha \bar{V}_{2}^{-\alpha-1}[n] V_{2}[n]-3 \bar{V}_{3}^{-2}[n]+2 \bar{V}_{3}^{-3}[n] V_{3}[n]\right] \\
& \triangleq \mathcal{S}_{y}\left(\bar{V}_{2}[n], \bar{V}_{3}[n], V_{2}[n], V_{3}[n]\right),
\end{aligned}
$$

where $\bar{V}_{2}[n]$ and $\bar{V}_{3}[n]$ are the given local points.

In the other case when $Y_{e}[n]<0, \mathcal{F}_{y}[n]$ can be approximated by its upper-bound function concerning $v_{2}[n]$ and $v_{3}[n]$. Specifically, it is transformed into a convex one as

$$
\begin{aligned}
& \mathcal{F}_{y}[n] \leq\left(2+\frac{\alpha}{2}\right) Y_{e}[n] \bar{v}_{2}^{-\frac{\alpha}{2}}[n] \bar{v}_{3}^{-1}[n] \\
& -\frac{\alpha}{2} Y_{e}[n] \bar{v}_{3}^{-1}[n] \bar{v}_{2}^{-\frac{\alpha}{2}-1}[n] v_{2}[n]-Y_{e}[n] \bar{v}_{2}^{-\frac{\alpha}{2}}[n] \bar{v}_{3}^{-2}[n] v_{3}[n] \\
& \triangleq \mathcal{Q}_{y}\left(\bar{v}_{2}[n], \bar{v}_{3}[n], v_{2}[n], v_{3}[n]\right),
\end{aligned}
$$

where $\bar{v}_{2}[n]$ and $\bar{v}_{3}[n]$ denote the given feasible points.

Using $\mathcal{I}_{y}[n]=1$ to imply $Y_{e}[n] \geq 0, E[n]$ can be 
approximated as

$$
\begin{aligned}
E[n] & =\mathcal{F}_{x}[n]+\mathcal{F}_{y}[n]+\mathcal{F}_{z}[n] \\
& \leq \mathcal{S}_{x}[n]+\mathcal{S}_{z}[n]+\mathcal{I}_{y}[n] \mathcal{S}_{y}[n]+\left(1-\mathcal{I}_{y}[n]\right) \mathcal{Q}_{y}[n] \\
& \triangleq \widetilde{E}[n] .
\end{aligned}
$$

After the aforementioned transformations, the original constraint (32c) can be rewritten as

$$
1+\frac{\rho}{\sigma^{2}} \widetilde{E}[n] \leq e^{\bar{t}_{2}[n]}\left(t_{2}[n]-\bar{t}_{2}[n]+1\right),
$$

which is convex now.

As a result, the trajectory optimization problem can be recasted into a convex problem as

$$
\begin{aligned}
& \max _{\substack{\mathbf{Q}, \mathbf{t}_{1}, \mathbf{t}_{2}, \mathbf{v}_{1}, \mathbf{v}_{2}, \mathbf{v}_{3} \\
\mathbf{V}_{1}, \mathbf{V}_{2}, \mathbf{V}_{3}}} \frac{1}{N} \sum_{n=1}^{N} t_{1}[n]-t_{2}[n] \\
& \text { s.t. } 1+\frac{\rho}{\sigma^{2}} \widetilde{D}[n] \geq e^{t_{1}[n]}, \\
& 1+\frac{\rho}{\sigma^{2}} \widetilde{E}[n] \leq e^{\bar{t}_{2}[n]}\left(t_{2}[n]-\bar{t}_{2}[n]+1\right), \\
& d_{u, l}^{2}[n]+\bar{v}_{1}^{2}[n]-2 \bar{v}_{1}[n] v_{1}[n] \leq 0, \forall n, \\
& d_{u, e}^{2}[n]+\bar{v}_{2}^{2}[n]-2 \bar{v}_{2}[n] v_{2}[n] \leq 0, \forall n, \\
& d_{U I}^{2}[n]+\bar{v}_{3}^{2}[n]-2 \bar{v}_{3}[n] v_{3}[n] \leq 0, \forall n \text {. } \\
& V_{1}^{2}[n]+\left\|\mathbf{q}^{r}[n]\right\|^{2}-2\left(\mathbf{q}^{r}[n]-\mathbf{C}_{l}\right)^{T} \mathbf{q}[n] \\
& -\left\|\mathbf{C}_{l}\right\|^{2}+H_{u}^{2} \leq 0 \text {, } \\
& V_{2}^{2}[n]+\left\|\mathbf{q}^{r}[n]\right\|^{2}-2\left(\mathbf{q}^{r}[n]-\mathbf{C}_{e}\right)^{T} \mathbf{q}[n] \\
& -\left\|\mathbf{C}_{e}\right\|^{2}+H_{u}^{2} \leq 0 \text {, } \\
& V_{3}^{2}[n]+\left\|\mathbf{q}^{r}[n]\right\|^{2}-2\left(\mathbf{q}^{r}[n]-\mathbf{C}_{I}\right)^{T} \mathbf{q}[n] \\
& -\left\|\mathbf{C}_{I}\right\|^{2}+H_{u I}^{2} \leq 0 \text {, } \\
& \mathbf{q}[1]=\mathbf{q}_{I},\left\|\mathbf{q}[N]-\mathbf{q}_{F}\right\|^{2} \leq\left(\frac{V_{m} T}{N}\right)^{2}, \\
& \|\mathbf{q}[n+1]-\mathbf{q}[n]\|^{2} \leq\left(\frac{V_{m} T}{N}\right)^{2}, n=1, \ldots, N-1,
\end{aligned}
$$

which can be solved efficiently by existing optimization tools. Due to the approximate conversions that we adopt above, the optimal solution to (65) is not equivalent to the problem (27). Hence, (65) needs to be solved iteratively to approach the optimal solution to (27), and the approximations will be compact if it converges.

\section{Overall Algorithm}

An effective algorithm is proposed to further enhance the network security by solving the three sub-problems alternately until convergence. With fixed UAV trajectory and IRS phase shifts, the optimal transmit beamforming can be obtained directly, while the solutions to the passive beamforming and UAV trajectory are updated by solving the approximate problems converted from the non-convex ones in each iteration. The detailed procedure of the iterative algorithm is summarized in Algorithm $1^{2}$.

\footnotetext{
${ }^{2}$ Using the iterative algorithm and SCA, the proposed design can be extended to a more general case of multiple legitimate users and eavesdroppers.
}

$\overline{\text { Algorithm } 1 \text { Iterative Algorithm for Joint Precoding and }}$ Trajectory Optimization

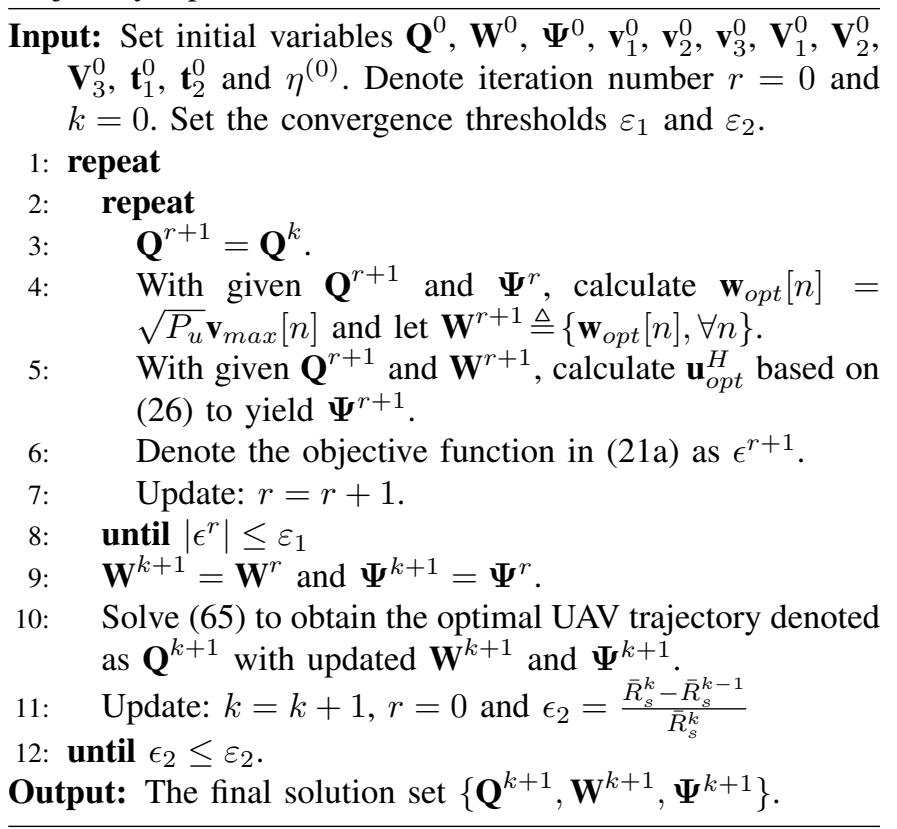

It can be noticed that there are two layers in Algorithm 1, including an outer iteration and an inner iteration. According to the results in [36], the fractional programming in the inner layer can always converge to the stationary and optimal solution. In the outer layer, the block coordinate descent method is adopted, and we update $\mathbf{Q}^{k}$ and $\left\{\mathbf{W}^{k}, \Psi^{k}\right\}$ in an alternating manner. Although the approximate problem for UAV trajectory optimization is solved in each iteration, the original objective value $\bar{R}_{s}$ is still non-decreasing over iterations due to the optimality of convex problems in each iteration. Furthermore, the objective value $\bar{R}_{s}$ by solving (25) or (65) generally gives a lower bound for the problem (15). As the objective value of the problem (15) is upper bounded by a finite value, the proposed algorithm is guaranteed to converge to at least a locally optimal solution based on the studies in [38]. Due to the closed-form solution to the active and passive beamforming in each iteration, the complexity of calculating $\mathbf{W}$ in Step 4 and obtaining $\Psi$ in Step 5 can be neglected compared to that of solving (65) in Step 10. Consequently, the complexity of the proposed algorithm is in the order of $\mathcal{O}\left(K N^{3.5}\right)$, where $K$ represents the number of iterations.

\section{Simulation Results}

In this section, simulations are conducted to evaluate the performance of the proposed design for secure IRS-assisted UAV networks. Unless stated otherwise, the default simulation parameters are presented in Table I. The coordinate system is set with the origin at the location of IRS. The legitimate user is assumed to be located at $\mathbf{C}_{l}=[20,30]^{T} \mathrm{~m}$, and the initial and final horizontal coordinates of UAV are set as $\mathbf{q}_{I}=[-300,80]^{T} \mathrm{~m}$ and $\mathbf{q}_{F}=[300,80]^{T} \mathrm{~m}$, respectively. Moreover, the UAV and the legitimate user are supposed to be in the same side of the RIS during the service to achieve effective reflection. The eavesdropper is randomly generated 
TABLE I

SimULATION PARAMETERS

\begin{tabular}{l|l}
\hline \hline UAV flight altitude & $H_{u}=100 \mathrm{~m}$ \\
\hline Maximum UAV velocity & $V_{m}=20 \mathrm{~m} / \mathrm{s}$ \\
\hline The altitude of IRS & $H_{I}=30 \mathrm{~m}$ \\
\hline Number of antennas & $N_{u}=16$ \\
\hline Number of IRS reflecting elements & $M=64$ \\
\hline Maximum transmit power & $P_{u}=20 \mathrm{dBm}$ \\
\hline $\begin{array}{l}\text { Channel power gain at the reference } \\
\text { distance }\end{array}$ & $\rho_{0}=-30 \mathrm{~dB}$ \\
\hline Noise power & $\sigma_{l}^{2}=\sigma_{e}^{2}=-80 \mathrm{dBm}$ \\
\hline Path loss exponents & $\alpha=3.5, \beta=2.4$ \\
\hline Rician factor & $\mathcal{K}=5$ \\
\hline Antenna separation & $\widetilde{d}=\frac{\lambda}{2}$ \\
\hline Element spacing of IRS & $\widetilde{d}_{x}=\widetilde{d}_{z}=\frac{\lambda}{4}$ \\
\hline
\end{tabular}

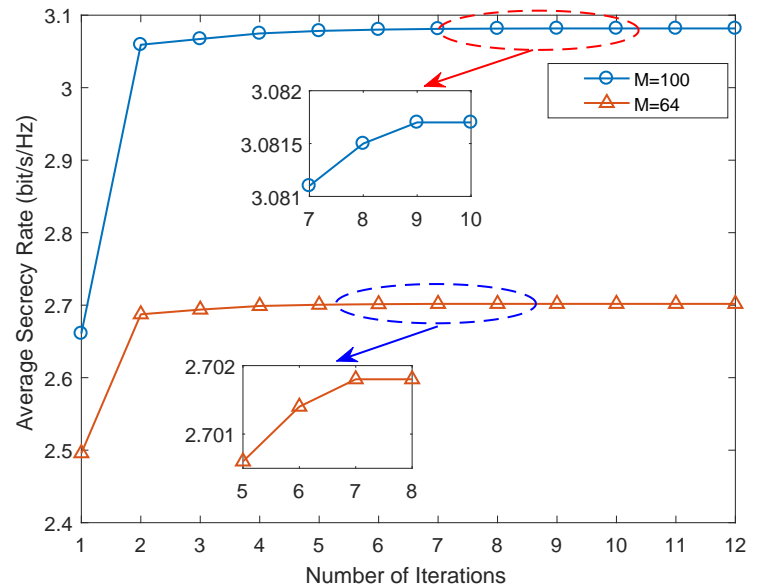

Fig. 2. Convergence of the proposed algorithm. $T=40 \mathrm{~s}$.

and located close to the legitimate user. In the simulations, we use the time slot length $\delta=T / N=1 \mathrm{~s}$ when designing the UAV's trajectory.

First, the convergence of the proposed algorithm is validated by numerical results as shown in Fig. 2 . We set $T=40 \mathrm{~s}$ and $N=40$. It can be observed that the iterative algorithm can converge quickly within 10 iterations for both $M=64$ and $M=100$. In addition, the average secrecy rate of $M=100$ is much higher than that of $M=64$.

Fig. 3 illustrates the distribution of the legitimate and eavesdropping users, and plots the optimal trajectories of UAV under different cases. The benchmark that jointly optimizes the transmit beamforming and UAV's trajectory without employing IRS is referred to as "Without IRS". With the given initial and final locations, one can see that $T=30 \mathrm{~s}$ is the minimum time required for the UAV to fly straight from the start to the destination at its maximum speed. For the case without IRS, it is shown that the UAV tends to be away from the eavesdropper to avoid being wiretapped. Due to the fact that the eavesdropper is near the legitimate user, the UAV is also far from the user.

In contrast, for the cases with $M=64$, the UAV flies rapidly at the initial and final stages while spending most of the

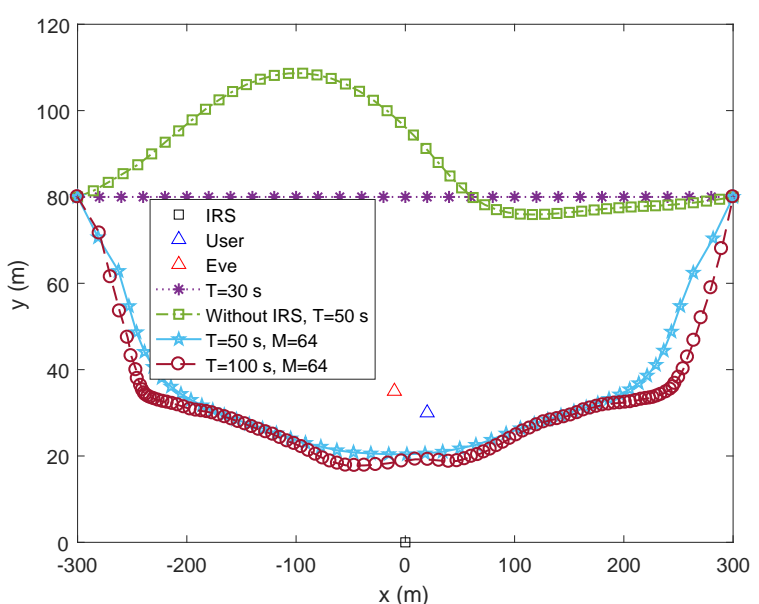

Fig. 3. The trajectories of UAV in different cases of the proposed scheme and the benchmark without IRS.

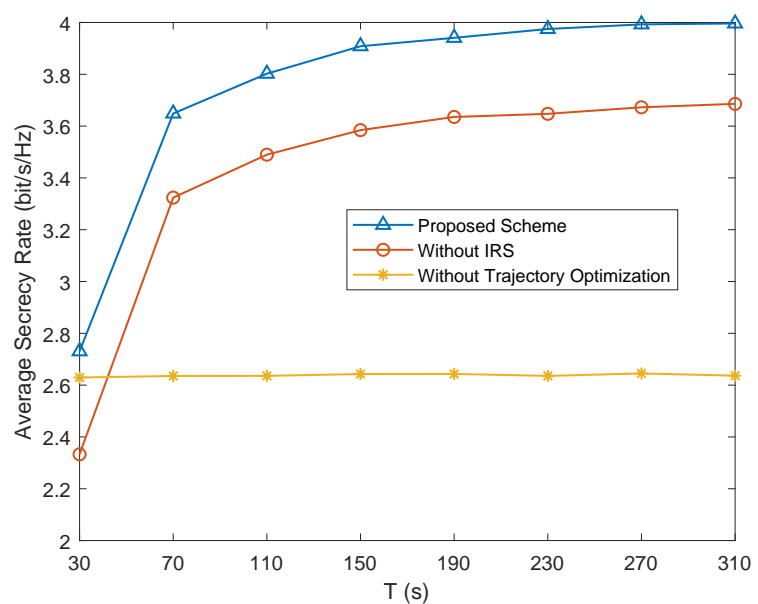

Fig. 4. Average secrecy rate versus the flying period $T$ of the proposed scheme and two benchmark schemes.

period flying slowly between the users and the IRS to improve the strengths of both the direct and reflecting channels. In addition, the UAV's speed is slower when $T=100 \mathrm{~s}$ compared with the case of $T=50 \mathrm{~s}$, to spend more time between them. This is because the benefit of the joint active and passive beamforming can be better reaped when the UAV flies between the user and the IRS.

In Fig. 4, we compare the proposed scheme with the other two benchmarks regarding the average secrecy rate when considering different $T$. The benchmark referred to as "Without Trajectory Optimization" performs the active and the passive beamforming design without optimizing the UAV's trajectory. From the results, one can see that the secrecy rate of both the proposed scheme and the optimization scheme without IRS (passive beamforming) increases with $T$ but gradually becomes saturated. The reason is that with larger $T$ the UAV can have more time to stay near the user and the IRS to provide better service for the legitimate user while combating the eavesdropping. The secrecy rate is stable with different values of $T$ if no trajectory optimization is applied, due to the fact that the UAV moves uniformly between the start and the end in a 


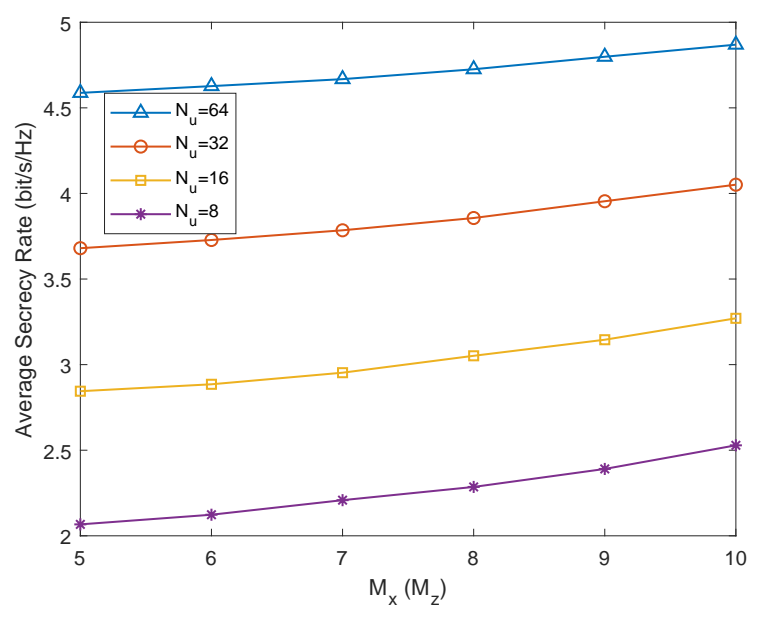

Fig. 5. Average secrecy rate for the proposed scheme with different number of reflecting elements and antennas. $T=50 \mathrm{~s}$.

straight line. More importantly, the superiority of the proposed scheme is demonstrated by the performance gap with the other two benchmarks. Clearly, the IRS can improve the secrecy rate by around 8 to 15 percent compared to the case without IRS. In addition, a considerable performance improvement can be found compared with the case without trajectory optimization. Therefore, both the trajectory optimization of UAV and passive beamforming of IRS play a significant role in enhancing the secure transmission.

Furthermore, we plot the average secrecy rate versus the number of reflecting elements in Fig. 5. Recall that $M=$ $M_{x} M_{z}$ and we have $M_{x}=M_{z}$ with a square IRS employed. As expected, the results show that the average secrecy rate increases with $M_{x}\left(M_{z}\right)$. This is because with a larger size of IRS, the passive beamforming gain of the legitimate user is able to be more benefited without resulting in the improvement of the eavesdropping power. By comparing the curves with different number of transmit antennas, we can conclude that equipping more antennas at the UAV also contributes to the security performance for the IRS-assisted UAV transmission. Thus, jointly deploying large-scale antennas and reflecting elements can provide more resource for active and passive beamforming to achieve a higher secrecy rate.

To gain more insight, we compare the proposed scheme with a legitimate rate maximization scheme marked by $\operatorname{Max} \bar{R}_{L}$ in Fig. 6 and Fig. 7, where the presented scheme is denoted by Max $\bar{R}_{s}$. Specifically, Fig. 6 plots the average achievable rate of the legitimate user and that of the eavesdropper versus the transmit power $P_{u}$ with $N_{u}=16$ and $N_{u}=32$. It is shown that the average achievable rate of the legitimate user, $\bar{R}_{L}$, increases with $P_{u}$ for both the two schemes. For the average achievable rate of the eavesdropper, $\bar{R}_{E}$, it grows with rising $P_{u}$ for the case Max $\bar{R}_{L}$ but keeps unchanged and is close to zero for the case Max $\bar{R}_{s}$. As expected, the legitimate rate maximization scheme achieves a little higher legitimate rate than the secrecy rate maximization scheme due to their respective objective functions, and the gap enlarges with $P_{u}$. By comparison, the performance of eavesdropping rate $\bar{R}_{E}$ differs significantly between them, and the gap tends to be

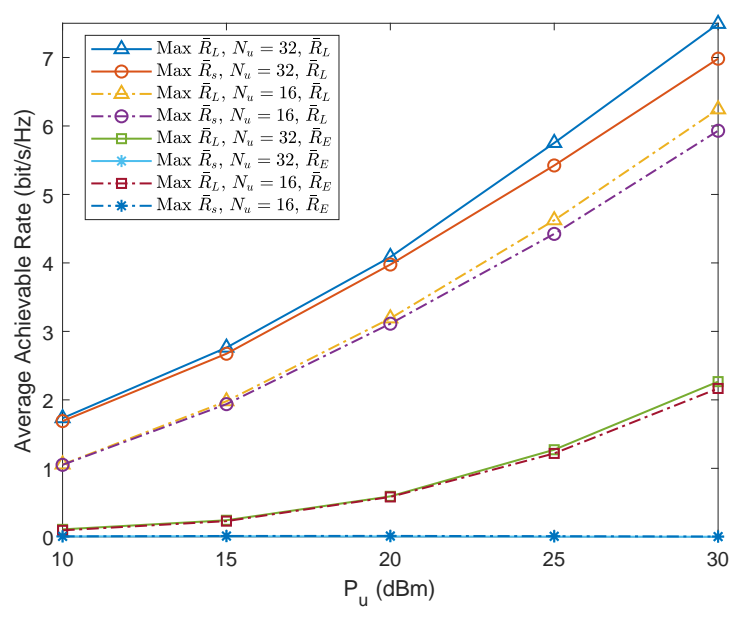

Fig. 6. Performance comparison of the legitimate user and the eavesdropper between the proposed scheme and the legitimate rate maximization scheme. $T=50 \mathrm{~s}$

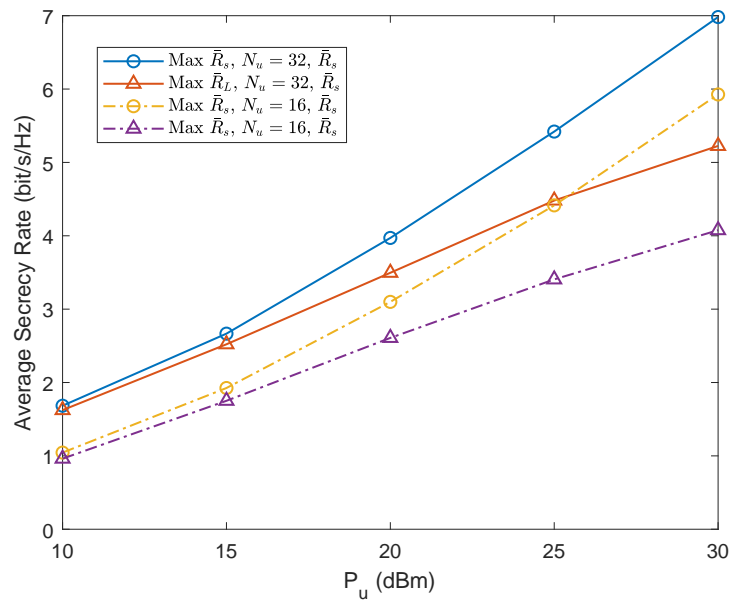

Fig. 7. Average secrecy rate versus the transmit power for the proposed scheme and the legitimate rate maximization scheme. $T=50 \mathrm{~s}$.

more prominent when $P_{u}$ becomes higher. The much lower value of $\bar{R}_{E}$ for Max $\bar{R}_{s}$ demonstrates that the proposed joint optimization can effectively suppress the eavesdropping and prevent information leakage. Besides, the results show that equipping more antennas is an effective way to improve the value of $\bar{R}_{L}$, whereas it does not affect $\bar{R}_{E}$.

In Fig. 7, the average secrecy rate $\bar{R}_{s}$ is compared for the two schemes. Obviously, $\bar{R}_{s}$ gets higher as $P_{u}$ becomes larger for all the cases. Moreover, we can see that the proposed scheme consistently outperforms the legitimate rate maximization scheme, and the gap between them enlarges with increasing $P_{u}$. Although the two schemes have similar $\bar{R}_{L}$ which increases with $P_{u}, \bar{R}_{E}$ is invariant for the case of Max $\bar{R}_{s}$ but grows for the case of Max $\bar{R}_{L}$ as in Fig. 6. As a result, the rising of $\bar{R}_{s}$ caused by increasing $P_{u}$ is more evident under the proposed scheme than the other. In addition, the average secrecy rate can be enhanced significantly by increasing the number of antennas from 16 to 32 , which is also consistent with the results in Fig. 6 .

To further investigate the influence of IRS, we plot the 


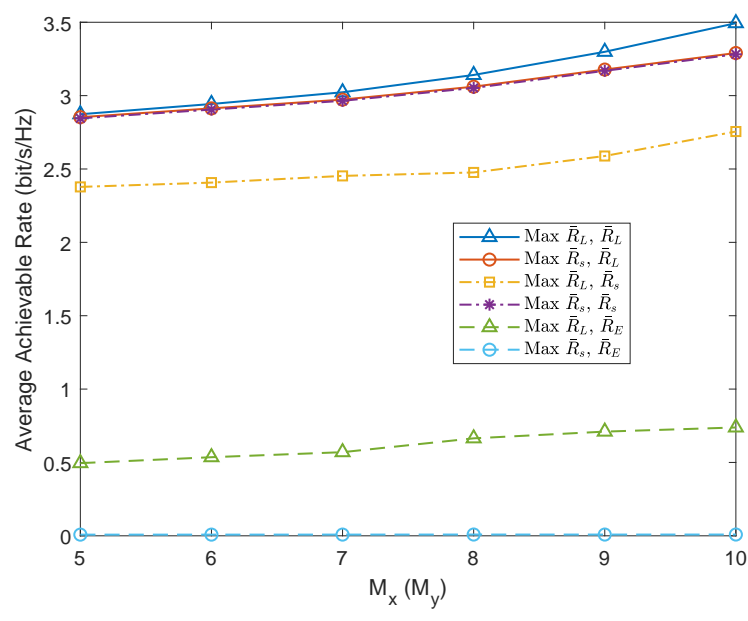

Fig. 8. Rate performance of the proposed scheme and the legitimate rate maximization scheme under different number of reflecting elements. $T=50$ s.

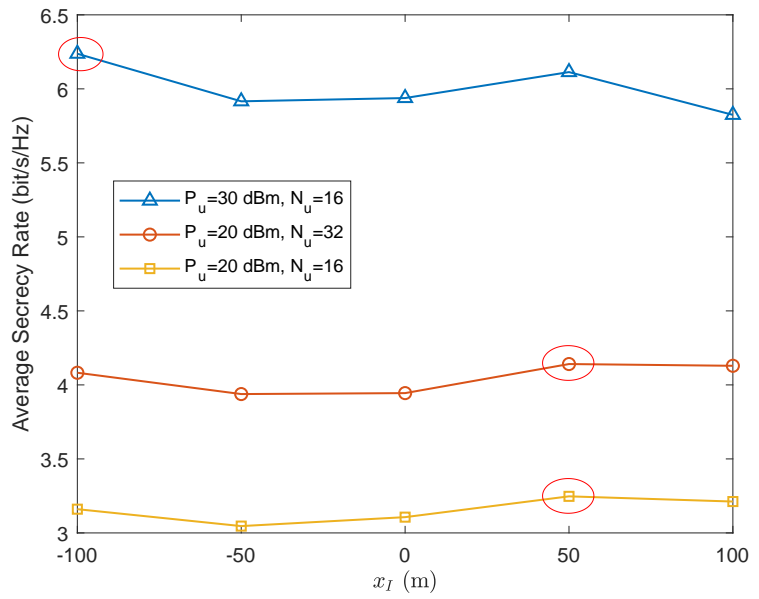

Fig. 9. Average secrecy rate versus the location of IRS along the $x$-axis. $y_{I}=0$ and $T=50 \mathrm{~s}$.

average achievable rate against the number of reflecting elements in Fig. 8. Specifically, we compare the proposed scheme with the benchmark scheme that aims at maximizing $\bar{R}_{L}$. It is observed that both $\bar{R}_{s}$ and $\bar{R}_{L}$ increase with equipping more reflecting elements for the two compared schemes. It is the same case for $\bar{R}_{E}$ with the objective Max $\bar{R}_{L}$, whereas $\bar{R}_{E}$ of the proposed scheme does not vary with the number of reflecting elements. One can see that the legitimate rate maximization scheme achieves similar $\bar{R}_{L}$ to the proposed scheme with a relatively small number of reflecting elements. Then, the former is higher than the latter with larger $M$. In terms of the average secrecy rate, there is a large performance gap between the proposed scheme and the benchmark, which can be explained by the tiny gap of $\bar{R}_{L}$ and the significant gap of $\bar{R}_{E}$. According to the results, the proposed secrecy transmission design is valid for all sizes of IRS and is more beneficial to the secure UAV transmission when deploying a large number of reflecting elements.

In Fig. 9, we intend to study the impact of IRS placement on the security performance. Following the coordinate system of Fig. 3, we fix the $y$-coordinate of IRS as $y_{I}=0$ and plot the average secrecy rate versus the $x$-coordinate of IRS. It can be seen that the location of IRS has an effect on the average secrecy rate, $\bar{R}_{s}$. The results show that $\bar{R}_{s}$ does not increase or decrease monotonically with IRS's $x$-coordinate. The value of $\bar{R}_{s}$ fluctuates more markedly under the case $P_{u}=30 \mathrm{dBm}$ than the other two cases with $P_{u}=20 \mathrm{dBm}$, due to the higher available power. In addition, one can see that $x_{I}=50 \mathrm{~m}$ achieves the optimal secrecy performance among the considered locations in Fig. 9 for the two cases of $P_{u}=20 \mathrm{dBm}$. This is mainly because the distance between the legitimate user and the IRS is relatively short, leading to a favorable reflecting channel. On the other hand, the eavesdropping channel is worse than the legitimate channel and the legitimate user can reap more beamforming gain. For the case of $P_{u}=30 \mathrm{dBm}$, the value of $\bar{R}_{s}$ is the highest when $x_{I}=-100 \mathrm{~m}$, to avoid the information leakage caused by the improved transmit power. Thus, the results motivate us to deploy the IRS at a more suitable location based on the practical conditions, such as the locations of users and the antenna and power resource.

\section{CONCLUSiOnS AND Future WORK}

In this paper, the secrecy rate maximization problem has been studied for IRS-assisted UAV wireless networks. To exploit the mobility of UAV and the beamforming gain of antennas and reflecting elements, we jointly optimize the transmit beamforming and trajectory of UAV and the passive beamforming of IRS. Due to the fact that the joint optimization problem is non-convex and intractable, we decompose it into three sub-problems and propose an iterative algorithm to solve them alternately. Specifically, the active beamforming vectors for UAV can be obtained directly. Then, the phase shift optimization problem which pertains to fractional programming is converted into a set of sub-problems in a parametric subtractive form. On this basis, we proceed to handle the UAV trajectory optimization problem, which is non-convex thereby approximated by a convex problem. The effectiveness of the proposed algorithm is demonstrated by the numerical results. It is worth noticing that several challenges, such as the endurance and stability of UAV and the accurate channel estimation, need to be resolved in the joint design. Moreover, the proposed scheme can be extended to a more general scenario with multiple legitimate and eavesdropping users after adopting proper multi-access methods and some imperative transformations. In our future work, robust and secure designs for IRS-assisted UAV networks considering multiple users and imperfect eavesdropping CSI will be investigated.

\section{REFERENCES}

[1] X. Pang, N. Zhao, J. Tang, D. Niyato, and K.-K. Wong, "Joint trajectory and beamforming optimization for secure UAV transmission aided by IRS," in Proc. IEEE WCSP'21, pp. 1-6, Changsha, China, Oct. 2021.

[2] Y. Zeng, R. Zhang, and T. J. Lim, "Wireless communications with unmanned aerial vehicles: Opportunities and challenges," IEEE Commun. Mag., vol. 54, no. 5, pp. 36-42, May 2016.

[3] Y. Zeng and R. Zhang, "Energy-efficient UAV communication with trajectory optimization," IEEE Trans. Wireless Commun., vol. 16, no. 6, pp. 3747-3760, Jun. 2017. 
[4] X. Jiang, M. Sheng, N. Zhao, C. Xing, W. Lu, and X. Wang, "Green UAV communications for 6G: A survey," Chinese J. Aeronaut., DOI: 10.1016/j.cja.2021.04.025.

[5] X. Pang, J. Tang, N. Zhao, X. Zhang, and Y. Qian, "Energy-efficient design for mmwave-enabled NOMA-UAV networks," Sci. China Inf. Sci., Apr. 2021, Art. no. 140303.

[6] Y. Zeng, J. Lyu, and R. Zhang, "Cellular-connected UAV: Potential, challenges, and promising technologies," IEEE Wireless Commun., vol. 26, no. 1, pp. 120-127, Feb. 2019.

[7] A. Al-Hourani, S. Kandeepan, and S. Lardner, "Optimal LAP altitude for maximum coverage," IEEE Wireless Commun. Lett., vol. 3, no. 6, pp. 569-572, Dec. 2014.

[8] M. Alzenad, A. El-Keyi, F. Lagum, and H. Yanikomeroglu, "3-D placement of an unmanned aerial vehicle base station (UAV-BS) for energyefficient maximal coverage," IEEE Wireless Commun. Lett., vol. 6, no. 4, pp. 434-437, Aug. 2017.

[9] M. Alzenad, A. El-Keyi, and H. Yanikomeroglu, "3-D placement of an unmanned aerial vehicle base station for maximum coverage of users with different QoS requirements," IEEE Wireless Commun. Lett., vol. 7, no. 1, pp. 38-41, Feb. 2018.

[10] J. Xu, Y. Zeng, and R. Zhang, "UAV-enabled wireless power transfer: Trajectory design and energy optimization," IEEE Trans. Wireless Commun., vol. 17, no. 8, pp. 5092-5106, Aug. 2018.

[11] Q. Wu, Y. Zeng, and R. Zhang, "Joint trajectory and communication design for multi-UAV enabled wireless networks," IEEE Trans. Wireless Commun., vol. 17, no. 3, pp. 2109-2121, Jan. 2018.

[12] N. Zhao, X. Pang, Z. Li, Y. Chen, F. Li, Z. Ding, and M. Alouini, "Joint trajectory and precoding optimization for UAV-assisted NOMA networks," IEEE Trans. Commun., vol. 67, no. 5, pp. 3723-3735, May 2019.

[13] C. Shen, T. Chang, J. Gong, Y. Zeng, and R. Zhang, "Multi-UAV interference coordination via joint trajectory and power control," IEEE Trans. Signal Process., vol. 68, pp. 843-858, Jan. 2020.

[14] J. Yao and J. Xu, "Joint 3D maneuver and power adaptation for secure UAV communication with CoMP reception," IEEE Trans. Wireless Commun., vol. 19, no. 10, pp. 6992-7006, Oct. 2020.

[15] M. Mozaffari, W. Saad, M. Bennis, Y. H. Nam, and M. Debbah, "A tutorial on UAVs for wireless networks: Applications, challenges, and open problems," IEEE Comm. Surveys Tuts., vol. 21, no. 3, pp. 2334 2360, 3rd Quart. 2019.

[16] R. Li, Z. Wei, L. Yang, D. W. K. Ng, J. Yuan, and J. An, "Resource allocation for secure multi-UAV communication systems with multieavesdropper," IEEE Trans. Commun., vol. 68, no. 7, pp. 4490-4506, Jul. 2020.

[17] Y. Cai, Z. Wei, R. Li, D. W. K. Ng, and J. Yuan, "Joint trajectory and resource allocation design for energy-efficient secure UAV communication systems," IEEE Trans. Commun., vol. 68, no. 7, pp. 4536-4553, Jul. 2020.

[18] X. Chen, D. Li, Z. Yang, Y. Chen, N. Zhao, Z. Ding, and F. R. Yu, "Securing aerial-ground transmission for NOMA-UAV networks," IEEE Netw., vol. 34, no. 6, pp. 171-177, Nov. 2020.

[19] Q. Wu and R. Zhang, "Towards smart and reconfigurable environment: Intelligent reflecting surface aided wireless network," IEEE Commun. Mag., vol. 58, no. 1, pp. 106-112, Jan. 2020.

[20] Q. Wu, S. Zhang, B. Zheng, C. You, and R. Zhang, "Intelligent reflecting surface aided wireless communications: A tutorial," IEEE Trans. Commun., vol. 69, no. 5, pp. 3313-3351, May 2021.

[21] Q. Wu and R. Zhang, "Intelligent reflecting surface enhanced wireless network via joint active and passive beamforming," IEEE Trans. Wireless Commun., vol. 18, no. 11, pp. 5394-5409, Nov. 2019.

[22] C. Huang, A. Zappone, G. C. Alexandropoulos, M. Debbah, and C. Yuen, "Reconfigurable intelligent surfaces for energy efficiency in wireless communication," IEEE Trans. Wireless Commun., vol. 18, no. 8, pp. 4157-4170, Aug. 2019.

[23] M. Cui, G. Zhang, and R. Zhang, "Secure wireless communication via intelligent reflecting surface," IEEE Wireless Commun. Lett., vol. 8, no. 5, pp. 1410-1414, Oct. 2019.

[24] S. Alfattani, W. Jaafar, Y. Hmamouche, H. Yanikomeroglu, A. Yonga çoglu, N. D. Dào, and P. Zhu, "Aerial platforms with reconfigurable smart surfaces for 5G and beyond," IEEE Commun. Mag., vol. 59, no. 1, pp. 96-102, Jan. 2021.

[25] X. Pang, M. Sheng, N. Zhao, J. Tang, D. Niyato, and K.-K. Wong, "When UAV meets IRS: Expanding air-ground networks via passive reflection," IEEE Wireless Commun., vol. 28, no. 5, pp. 164-170, Oct. 2021.

[26] S. Li, B. Duo, X. Yuan, Y. Liang, and M. Di Renzo, "Reconfigurable intelligent surface assisted UAV communication: Joint trajectory design and passive beamforming," IEEE Wireless Commun. Lett., vol. 9, no. 5, pp. 716-720, May 2020.

[27] L. Ge, P. Dong, H. Zhang, J. Wang, and X. You, "Joint beamforming and trajectory optimization for intelligent reflecting surfaces-assisted UAV communications," IEEE Access, vol. 8, pp. 78702-78712, 2020.

[28] Z. Wei, Y. Cai, Z. Sun, D. W. K. Ng, J. Yuan, M. Zhou, and L. Sun, "Sum-rate maximization for IRS-assisted UAV OFDMA communication systems," IEEE Trans.. Wireless Commun., vol. 20, no. 4, pp. 2530 2550, Apr. 2021.

[29] S. Fang, G. Chen, and Y. Li, "Joint optimization for secure intelligent reflecting surface assisted UAV networks," IEEE Wireless Commun. Lett., vol. 10, no. 2, pp. 276-280, Feb. 2021.

[30] Z. Mohamed and S. Aissa, "Leveraging UAVs with intelligent reflecting surfaces for energy-efficient communications with cell-edge users," in Proc. IEEE 2020 ICC Workshops, pp. 1-6, Dublin, Ireland, Jun. 2020.

[31] Q. Zhang, W. Saad, and M. Bennis, "Reflections in the sky: Millimeter wave communication with UAV-carried intelligent reflectors," in Proc. IEEE GLOBECOM'17, pp. 1-6, Waikoloa, HI, USA, Dec. 2019.

[32] S. Jiao, F. Fang, X. Zhou, and H. Zhang, "Joint beamforming and phase shift design in downlink UAV networks with IRS-assisted NOMA," $J$. Commun. Inf. Netw., vol. 5, no. 2, pp. 138-149, Jun. 2020

[33] H. Lu, Y. Zeng, S. Jin, and R. Zhang, "Aerial intelligent reflecting surface: Joint placement and passive beamforming design with 3D beam flattening," IEEE Trans. Wireless Commun., vol. 20, no. 7, pp. 4128 4143, Jul. 2021.

[34] C. Hu, L. Dai, S. Han, and X. Wang, "Two-timescale channel estimation for reconfigurable intelligent surface aided wireless communications," IEEE Trans. Commun., vol. 69, no. 11, pp. 7736-7747, Nov. 2021.

[35] Q. Li and W. Ma, "Optimal and robust transmit designs for MISO channel secrecy by semidefinite programming," IEEE Trans. Signal Process., vol. 59, no. 8, pp. 3799-3812, Aug. 2011.

[36] W. Dinkelbach, "On nonlinear fractional programming," Management Science, vol. 13, no. 7, pp. 492-498, Mar. 1967.

[37] Y. Sun, P. Babu, and D. P. Palomar, "Majorization-minimization algorithms in signal processing, communications, and machine learning," IEEE Trans. Signal Process., vol. 65, no. 3, pp. 794-816, Feb. 2017.

[38] M. Hong, M. Razaviyayn, Z. Luo, and J. Pang, "A unified algorithmic framework for block-structured optimization involving big data: With applications in machine learning and signal processing," IEEE Signal Process. Mag., vol. 33, no. 1, pp. 57-77, Jan. 2016.

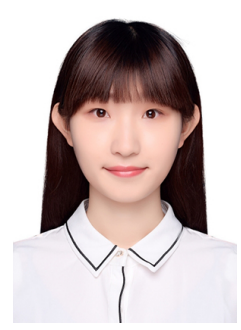

Xiaowei Pang received the B.S. degree in communication engineering from Northwestern Polytechnical University, Xi'an, China, in 2018. She is currently working toward the PhD degree in the School of Information and Communication Engineering, Dalian University of Technology, Dalian, China. Her current research interests include UAV communications, NOMA technique, intelligent reflecting surface, physical layer security, and convex optimization of wireless networks.

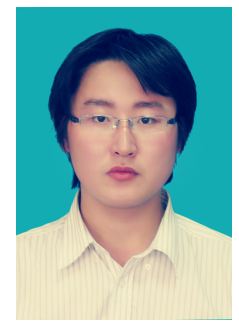

Nan Zhao (Senior Member, IEEE) is currently a Professor at Dalian University of Technology, China. $\mathrm{He}$ received the Ph.D. degree in information and communication engineering in 2011, from Harbin Institute of Technology, Harbin, China. Dr. Zhao is serving on the editorial boards of IEEE Wireless Communications and IEEE Wireless Communications Letters. He won the best paper awards in IEEE VTC 2017 Spring, ICNC 2018, WCSP 2018 and WCSP 2019. He also received the IEEE CommuYoung Researcher Award in 2018. 


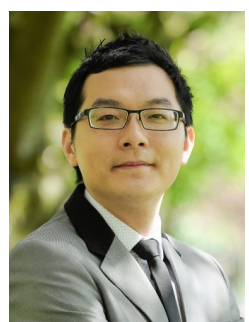

Jie Tang (Senior Member, IEEE) received the B.Eng. degree from South China University of Technology, China, the M.Sc. degree from the University of Bristol, UK, and the Ph.D. degree from Loughborough University, UK. From 2013 to 2015, he was a research associate at the School of Electrical and Electronic Engineering, University of Manchester, UK. He is now a professor at the School of Electronic and Information Engineering, South China University of Technology, China.

His current research interests include SWIPT, UAV communications, NOMA, reconfigurable intelligent surface. He is currently serving as an Editor for IEEE WIRELESS COMMUNICATIONS LETTERS, IEEE SYSTEMS JOURNAL, and IEEE ACCESS. He is the Guest Editor for two special issues in IEEE TRANSACTIONS ON GREEN COMMUNICATIONS AND NETWORKING, and one special issue in IEEE OPEN JOURNAL OF THE COMMUNICATIONS SOCIETY. He also served as a Track Co-Chair of IEEE VTC-Spring 2018, Symposium Co-Chair of IEEE/CIC ICCC 2020, IEEE ComComAp 2019, TPC Co-Chair of EAI GreeNets 2019, and Workshop Co-Chair of IEEE ICCC/CIC 2019. He is a co-recipient of the ICNC 2018, CSPS 2018, WCSP 2019 and 6GN 2020 Best Paper Awards. He is the current Vice Chair of the Special Interest Group on Green Cellular Networks within the IEEE ComSoc Green Communications and Computing Technical Committee.

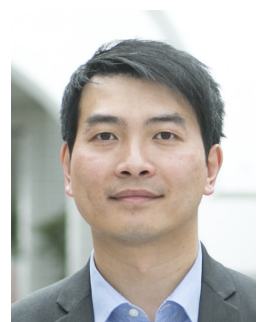

Kai-Kit Wong (Fellow, IEEE) received the BEng, the MPhil, and the PhD degrees, all in Electrical and Electronic Engineering, from the Hong Kong University of Science and Technology, Hong Kong, in 1996, 1998, and 2001, respectively. After graduation, he took up academic and research positions at the University of Hong Kong, Lucent Technologies, Bell-Labs, Holmdel, the Smart Antennas Research Group of Stanford University, and the University of Hull, UK. He is Chair in Wireless Communications at the Department of Electronic and Electrical Engineering, University College London, UK. His current research centers around 5G and beyond mobile communications. He is a co-recipient of the 2013 IEEE Signal Processing Letters Best Paper Award and the 2000 IEEE VTS Japan Chapter Award at the IEEE Vehicular Technology Conference in Japan in 2000, and a few other international best paper awards. He is Fellow of IEEE and IET and is also on the editorial board of several international journals. $\mathrm{He}$ is the Editor-in-Chief for IEEE Wireless Communications Letters since 2020 .

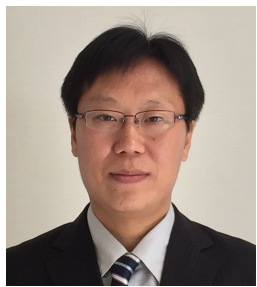

Celimuge Wu (Senior Member, IEEE) received his $\mathrm{PhD}$ degree from The University of ElectroCommunications, Japan in 2010. He has been an associate professor of The University of ElectroCommunications since 2015. His research interest$\mathrm{s}$ include Vehicular Networks, Edge Computing, IoT, Intelligent Transport Systems, and Application of Machine Learning in Wireless Networking and Computing. He serves as an associate editor of IEEE Transactions on Network Science and Engineering, IEEE Transactions on Green Communications and Networking, IEEE Open Journal of the Computer Society, Wireless Networks, and IEICE Transactions on Communications. He is the chair of IEEE TCGCC Special Interest Group on Green Internet of Vehicles and IEEE TCBD Special Interest Group on Big Data with Computational Intelligence. He is a recipient of 2021 IEEE Communications Society Outstanding Paper Award, 2021 IEEE Internet of Things Journal Best Paper Award, and IEEE Computer Society 2019 Best Paper Award Runner-Up. He is a senior member of IEEE.

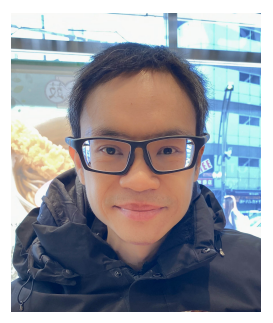

Dusit Niyato (Fellow, IEEE) is currently a professor in the School of Computer Science and Engineering, at Nanyang Technological University, Singapore. He received B.Eng. from King Mongkuts Institute of Technology Ladkrabang (KMITL), Thailand in 1999 and Ph.D. in Electrical and Computer Engineering from the University of Manitoba, Canada in 2008. His research interests are in the areas of Internet of Things (IoT), machine learning, and incentive mechanism design. 